\title{
Tune compensation in nearly scaling fixed field alternating gradient accelerators
}

\author{
M. Haj Tahar®* \\ CERN, Geneva 23, Switzerland \\ F. Méot \\ Collider Accelerator Department, Brookhaven National Laboratory (BNL), \\ Long Island, Upton, New York 11973, USA
}

(Received 12 September 2019; accepted 12 May 2020; published 22 May 2020)

\begin{abstract}
In this paper, we investigate the stability of the particle trajectories in fixed field alternating gradient accelerators (FFAs) in the presence of field errors. The emphasis is on the scaling radial sector FFA type: A collaboration work is ongoing in view of better understanding the properties of the $150 \mathrm{MeV}$ scaling FFA at Kyoto University Institute for Integrated Radiation and Nuclear Science in Japan and progress toward high-intensity operation. Analysis of certain types of field imperfections revealed some interesting features that required the development of an analytical model based on the scalloping angle of the orbits. This helped explain some of the experimental results as well as generalize the concept of a scaling FFA to a nonscaling one for which the tune variations obey a well-defined law. Based on this, a compensation scheme of tune variations in imperfect scaling FFAs is presented. This is the cornerstone of a novel concept of a fixed tune FFA in which the scaling is not achieved at every azimuthal position of the ring but rather in an average sense.
\end{abstract}

DOI: 10.1103/PhysRevAccelBeams.23.054003

\section{INTRODUCTION}

The scaling fixed field alternating gradient accelerator (FFA) is a concept that was invented in the 1950s almost independently in the United States, Japan, and USSR [1-3]. Several electron machines were built in the United States. However, it was not until the 1990s that the interest for scaling FFAs was revived in Japan. Several machines were built, among which is a $150 \mathrm{MeV}$ proton machine at Kyoto University Institute for Integrated Radiation and Nuclear Science (KURNS) [4]. One main feature of this machine is its potential for high-power applications, hence its use as a proton driver for an accelerator-driven subcritical reactor [5] at KURNS. A large dynamic acceptance can be achieved, since the crossing of the betatron resonances is avoided in this concept. This is achieved by introducing an $R^{k}$ increase of the magnetic field with the radius, resulting in the beam experiencing the same focusing throughout the acceleration, therefore keeping the tunes constant. The magnetic field profile allowing this is written in cylindrical coordinates in the form $B=B_{0}\left(R / R_{0}\right)^{k} F(\theta)$, where $B$ is

\footnotetext{
*hajtaham@gmail.com

Published by the American Physical Society under the terms of the Creative Commons Attribution 4.0 International license. Further distribution of this work must maintain attribution to the author(s) and the published article's title, journal citation, and DOI.
}

the vertical component of the magnetic field in the median plane, $R$ is the radial coordinate with respect to the center of the ring, $B_{0}$ is the reference field at $R=R_{0}, F(\theta)$ is the flutter function describing the azimuthal variations of the field, and $k$ is the average field index of the magnets, sometimes also referred to as the scaling factor, defined by $k=R / B . \partial B / \partial R$ and, ideally, a constant value everywhere in the ring. One shall insist here that this form of the magnetic field is a sufficient but non-necessary condition in order to obtain a fixed tune FFA. In the scaling FFA concept, the orbit excursion is uniquely determined by the field index and the flutter function. Given that all built machines in the past opted for a phase advance per cell below $180^{\circ}$, the orbit shift is therefore large, of the order of $1 \mathrm{~m}$, which makes the magnet larger than typical synchrotron magnets. Besides, due to the complexity of the field profile and flutter of the magnets, field imperfections can be problematic and difficult to cure, since the orbits move outward from the center of the machine with increasing energies. This can lead to the crossing of several betatron resonances at low speed and to beam deterioration in consequence. For instance, this is a strong contribution to the overall low beam transmission in the KURNS FFA $[6,7]$.

Despite some controversy regarding the definition of FFA, it is worth mentioning that spiral sector cyclotrons are a class of nonscaling FFA where the alternating gradient effect comes from the spiral-shaped poles [1]. Some of 
these machines existed since the 1950s such as the Paul Scherrer Institut and Tri-University Meson Facility cyclotrons which have been operating for over 40 years at the high-intensity regime with very low losses. In this concept, the scaling is sacrificed in favor of isochronism, thus allowing such machines to operate in the continuous wave mode. Unlike cyclotrons, scaling FFAs operate like synchrocyclotrons in that the rf frequency must be varied to remain synchronous with the accelerating particles.

In addition, although FFAs are generally assumed to have a nonlinear field profile with the radius, there exists a second class of FFAs, that of linear concepts. The linear FFAs are so called because they use only linear elements such as dipoles and quadrupoles. A proof-of-principle electron model of this concept called Electron Model for Many Applications [8] has been constructed and operated successfully at the Science and Technology Facilities Council Daresbury Laboratory in the United Kingdom. Such a concept has been proposed in the context of rapid acceleration of unstable muons for future high-energy colliders. Furthermore, the first energy recovery linac based on linear FFA magnets is being constructed at Cornell University [9] as a prototype for a potential electron ion collider, the so-called eRHIC [10].

In the original work of Symon [1], it was established that if the average field index is kept constant and the closed orbits are geometrically similar in a way that will become clearer later on in this paper, then the number of betatron oscillations can be approximated by

$$
\begin{aligned}
& \nu_{x}^{2} \approx k+1, \\
& \nu_{y}^{2} \approx-k+\mathcal{F}^{2}\left[1+2 \tan ^{2}(\xi)\right],
\end{aligned}
$$

where $\mathcal{F}$ is the magnetic flutter and $\xi$ is the spiral angle of the magnets. Nevertheless, due to field imperfections, it is particularly challenging to design and manufacture a magnet which produces exactly the desired magnetic field. For instance, the measured as well as the simulated tunes of the $150 \mathrm{MeV}$ FFA at KURNS are shown in Fig. 1, where one can observe non-negligible tune variations.

In what follows, we will derive the linearized Hill's equations of motion in cylindrical coordinates in order to take into account the field imperfections and define the domain of validity of the above approximation [Eq. (1)]. In particular, it will be shown that the linear motion around the planar closed orbit in a radial sector FFA is fully determined by two main parameters: the scalloping angle of the closed orbit and the average field index of the magnet. One objective of this paper is to establish approximate expressions of the ring tunes that take into account the nonscaling of the orbits due to field imperfections and compare with the tracking results. This will be a crucial result in order to establish a correction scheme to minimize the tune variations in imperfect radial scaling FFA.

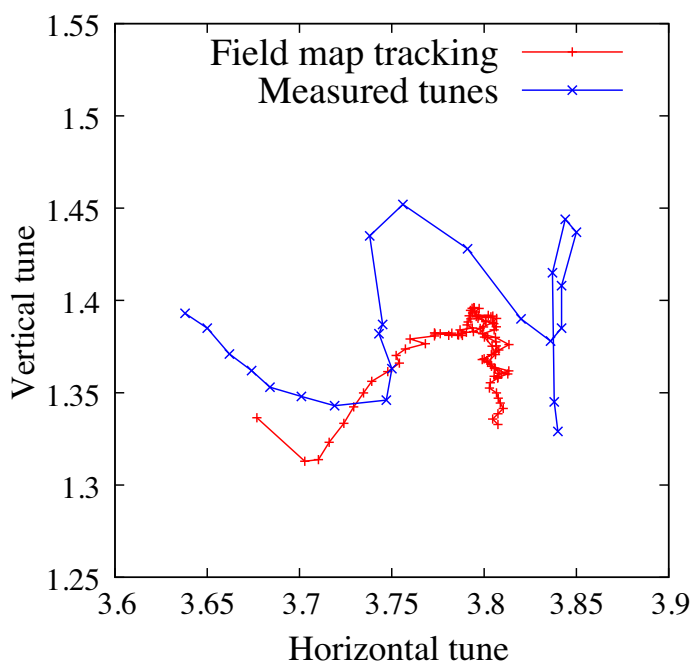

FIG. 1. Betatron tunes from 11 to $100 \mathrm{MeV}$ (left to right). The details of the measurement as well as the methods and tools that were used to characterize the $150 \mathrm{MeV}$ KURNS FFA are discussed in Ref. [7].

\section{GEOMETRY OF THE CLOSED ORBIT}

In cylindrical coordinates as shown in Fig. 2, it can be shown that any median plane closed orbit satisfies the following equation:

$$
\tan (\phi)=-\frac{d R / d \theta}{R}=-\frac{\dot{R}}{R}
$$

where $\phi(\theta)$ is the angle between the extended radial line and the normal to the equilibrium orbit, i.e., $\phi=\left(\widehat{\overrightarrow{u_{R}}, \overrightarrow{u_{x}}}\right)$.

$\phi$ is a natural parameter to describe the scalloping of the closed orbit (note that $\phi=-T$ for the element "polarmes" in the tracking code ZGOUBI [11]): If $\phi(\theta)=0$ for all $\theta$, then the closed orbit is a circle. In order for $R$ to be a welldefined function of the azimuthal angle, the orbit cannot be

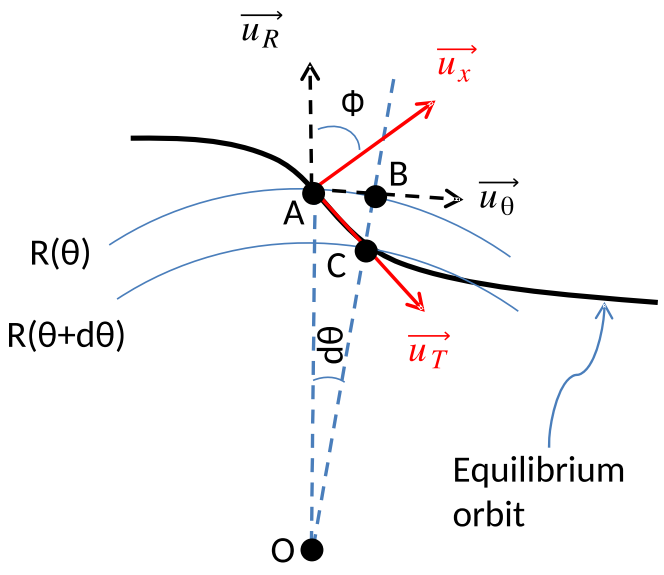

FIG. 2. Geometric properties of the closed orbit. The point $\mathrm{O}$ is the center of the ring, and the particle motion is clockwise. $\overrightarrow{u_{T}}$ is the unit vector along the momentum direction. 
moving radially outward. This sets the following condition on $\phi:|\phi|<\pi / 2$.

Differentiating again with respect to the azimuthal angle yields

$$
\ddot{R}=-\dot{R} \tan (\phi)-R \dot{\phi}\left[1+\tan ^{2}(\phi)\right] .
$$

Now, the signed curvature of the closed orbit can be expressed as a function of the radius:

$$
\frac{1}{\rho}=\frac{R^{2}+2(\dot{R})^{2}-R \ddot{R}}{\left[R^{2}+(\dot{R})^{2}\right]^{3 / 2}}=\frac{1}{R}(1+\dot{\phi}) \cos (\phi),
$$

where we use the convention that the particle motion is clockwise with increasing $\theta$ and that the curvature is positive if the momentum vector turns clockwise with increasing $\theta$.

Thus, when defining the particle closed orbit in cylindrical coordinates, the particle radius $R$ changes with the azimuthal angle. Such a dependence is described by the angle $\phi(\theta)$, which is also defined as the angle between the tangent to the circle of radius $R(\theta)$ and the particle momentum vector at the azimuthal angle $\theta$. It results that the position of the particle on the closed orbit is specified by the variables $[R(\theta), \theta, z(\theta)]$. In addition, the geometrical properties of each closed orbit will be periodic in the azimuthal angle $\theta$ with period $2 \pi / N, N$ being the number of identical sectors in the ring.

Note that, in a drift space where the particle trajectory is a straight line, Eq. (4) holds, and it can be easily shown that $\dot{\phi}=-1$ by observing that the vector $\overrightarrow{u_{x}}$ remains unchanged along a straight line. In addition, $(1+\dot{\phi})>0$ for positive curvature, which implies that the vertical component of the magnetic field points upward for positive charged particles.

\section{A. Arclength in cylindrical and generalized azimuthal coordinates}

To describe the transverse beam dynamics in particle accelerators, very often the first approach consists in searching for the closed orbit corresponding to a specific energy and writing the linearized Hill's equation of motion describing the betatron oscillations around that orbit. In the seminal Midwestern Universities Research Association paper of 1956, Symon introduced the concept of generalized azimuthal coordinates in which each orbit is specified by its equivalent radius $\mathcal{R}$ defined in the following way:

$$
\mathcal{C}=2 \pi \mathcal{R},
$$

where $\mathcal{C}$ is the closed orbit length for a specific energy. In addition, a generalized azimuthal coordinate is defined which is related to the distance measured along the orbit, i.e., to the curvilinear abscissa $s$, in the following way:

$$
s=\mathcal{R} \vartheta .
$$

In the formalism to be developed in the following section, the linearized equations of motion in cylindrical coordinates will be derived which are particularly useful when calculating a correction scheme of magnetic field imperfections. For this reason, we will express the increment $d s$ in $s$ as a function of the increment $d \theta$ in $\theta$ along a closed orbit.

The increment of the arclength in cylindrical coordinates is given by

$$
\begin{aligned}
d s & =\left(d R^{2}+R^{2} d \theta^{2}+d z^{2}\right)^{1 / 2} \\
& =R\left[1+\left(\frac{\dot{R}}{R}\right)^{2}+\left(\frac{\dot{z}}{R}\right)^{2}\right]^{1 / 2} d \theta .
\end{aligned}
$$

Neglecting the changes in the vertical direction (the ideal closed orbit usually lies in the median plane of the accelerator), then one obtains by making use of Eq. (2)

$$
\begin{gathered}
\frac{d s}{d \theta}=\frac{R(\theta)}{\cos (\phi)}, \\
\frac{d^{2} s}{d \theta^{2}}=\frac{\dot{R}}{\cos (\phi)}+\frac{R \dot{\phi} \tan (\phi)}{\cos (\phi)} .
\end{gathered}
$$

Note that Eq. (8) can be also established by basic trigonometric considerations in the triangle $O A C$ in Fig. 2 and by taking the limit $d \theta \rightarrow 0$. Equating the change of the arclength in both coordinates yields

$$
d s=\mathcal{R} d \vartheta=\frac{R}{\cos (\phi)} d \theta
$$

so that one can establish the transformation from the generalized azimuthal coordinate to the cylindrical azimuthal coordinate:

$$
d \vartheta=\frac{R}{\mathcal{R}} \frac{1}{\cos (\phi)} d \theta
$$

and

$$
\vartheta=\frac{1}{\mathcal{R}} \int_{0}^{\theta} \frac{R(u)}{\cos (\phi)} d u,
$$

where one assumed that both quantities coincide at $\theta=0$. In particular, this shows that, even for a radial sector FFA, the generalized azimuthal angle $\vartheta$ and the polar angle $\theta$ are not identical.

In addition, $\mathcal{R}$ can be defined in cylindrical coordinates as follows: 


$$
\mathcal{R}=\frac{\mathcal{C}}{2 \pi}=\frac{1}{2 \pi} \int_{0}^{2 \pi} \frac{R}{\cos (\phi)} d \theta
$$

which shows that $\mathcal{R}$ will be larger than the mean radius $\langle R\rangle$ of the closed orbit.

Now, by making use of Eqs. (4) and (10), we can compute the average value of the curvature function for a given closed orbit in the following way:

$$
\begin{aligned}
\frac{1}{2 \pi} \int_{0}^{2 \pi \mathcal{R}} \frac{d s}{\rho} & =\frac{1}{2 \pi} \int_{0}^{2 \pi} \frac{R(\theta)}{\rho(\theta)} \frac{1}{\cos (\phi)} d \theta \\
& =\frac{1}{2 \pi} \int_{0}^{2 \pi}(1+\dot{\phi}) d \theta=1
\end{aligned}
$$

as expected, since the sum of all deflecting angles of the closed orbit shall equal $2 \pi$.

\section{TRANSVERSE EQUATIONS OF MOTION IN CYLINDRICAL COORDINATES}

The transverse equations for the linear betatron oscillations around the closed orbit are written in the following way [12]:

$$
\begin{gathered}
\frac{d^{2} x}{d s^{2}}+\frac{1-n}{\rho^{2}} x=0, \\
\frac{d^{2} y}{d s^{2}}+\frac{n}{\rho^{2}} y=0,
\end{gathered}
$$

where $\rho$ is the signed curvature of the closed orbit which is a function of $s$ while $x$ and $y$ are the transverse deviations of the particle in the horizontal and vertical direction, respectively. Since the field map in a fixed field accelerator is usually defined in cylindrical coordinates $(R, \theta)$ with respect to the center of the ring, it is natural to write the particle equations of motion in such a frame. Differentiating with respect to the azimuthal angle $\theta$ yields

$$
\begin{gathered}
\frac{d x}{d \theta}=\frac{d x}{d s} \frac{d s}{d \theta}, \\
\frac{d^{2} x}{d \theta^{2}}=\frac{d^{2} x}{d s^{2}}\left(\frac{d s}{d \theta}\right)^{2}+\frac{d x}{d s} \frac{d^{2} s}{d \theta^{2}},
\end{gathered}
$$

where the transverse coordinate is a function of $\theta$ only. Now, injecting Eqs. (17) and (18) into the Hill's equations (15) and (16) yields

$$
\begin{gathered}
\ddot{x}-\frac{\ddot{s}}{\dot{s}} \dot{x}+\frac{\dot{s}^{2}}{\rho^{2}}(1-n) x=0, \\
\ddot{y}-\frac{\ddot{s}}{\dot{s}} \dot{y}+\frac{\dot{s}^{2}}{\rho^{2}} n y=0,
\end{gathered}
$$

where the $(\dot{)}$ represents the differentiation with respect to the azimuthal variable. The above equations can be written in the standard form:

$$
\ddot{u}+p(\theta) \dot{u}+q(\theta) u=0 .
$$

This is a second-order linear differential equation with variable coefficients, for which, in general, a closed form solution is not known. The first-order derivative is removed by making the Liouville-Green transformation:

$$
u(\theta)=v(\theta) \cdot \exp \left(-\frac{1}{2} \int_{\theta_{0}}^{\theta} p(h) d h\right)
$$

which yields

$$
\ddot{v}+\left(q(\theta)-\frac{1}{2} \frac{d p}{d \theta}-\frac{p^{2}}{4}\right) v=0
$$

Now, back to Eqs. (19) and (20). The above formalism applies by simply replacing $p(\theta)=-\ddot{s} / \dot{s}$, which yields

$$
u(\theta)=\frac{\sqrt{d s / d \theta}}{\sqrt{d s / d \theta_{\theta_{0}}}} v(\theta) \propto \sqrt{\frac{R(\theta)}{\cos (\phi)}} v(\theta),
$$

and the differential equations for the linear betatron oscillations around the planar equilibrium orbit are given by

$$
\begin{aligned}
& \ddot{v}_{x, y}+\left[q_{x, y}(\theta)+f(\theta)\right] v_{x, y}=0, \\
& q_{x}(\theta)=\frac{\dot{s}^{2}}{\rho^{2}}(1-n)=(1+\dot{\phi})^{2}(1-n), \\
& q_{y}(\theta)=\frac{\dot{s}^{2}}{\rho^{2}} n=(1+\dot{\phi})^{2} n, \\
& p(\theta)=-\frac{\ddot{s}}{\dot{s}}=(1-\dot{\phi}) \tan (\phi), \\
& f(\theta)=-\frac{\dot{p}}{2}-\frac{p^{2}}{4} .
\end{aligned}
$$

In the next section, we shall seek the expression of the field index in cylindrical coordinates.

\section{A. Field variations \\ 1. Field index}

Using the chain rule for partial differentiation, one obtains

$$
n=-\frac{\rho}{B} \frac{\partial B}{\partial x}=-\frac{\rho}{B}\left[\frac{\partial B}{\partial R} \frac{\partial R}{\partial x}+\frac{\partial B}{\partial \theta} \frac{\partial \theta}{\partial x}\right],
$$

where $B$ is the magnetic field seen by the particle.

Applying the law of sines in the triangles $A B D$ and $O A D$ as illustrated in Fig. 3, the following relations can be established [13]: 


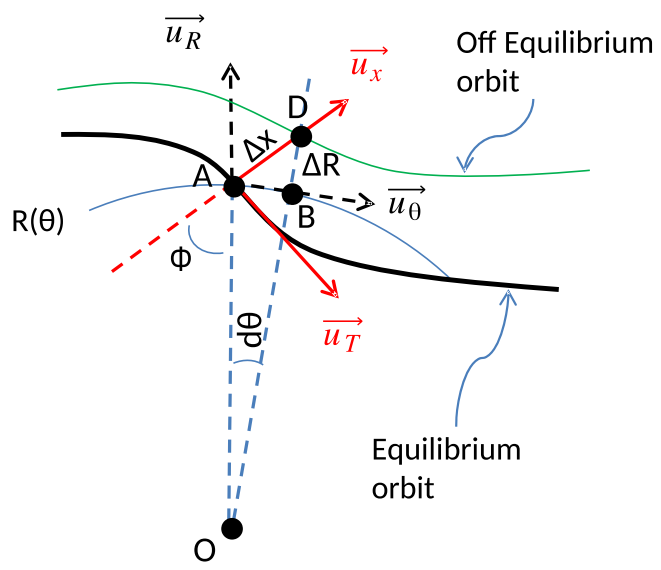

FIG. 3. Geometric properties of the particle motion around the closed orbit.

$$
\begin{aligned}
& \frac{\partial R}{\partial x}=\cos (\phi), \\
& \frac{\partial \theta}{\partial x}=\frac{\sin (\phi)}{R} .
\end{aligned}
$$

Next, we substitute Eqs. (4), (27), and (28) into Eq. (26) to get the expression of the field index:

$$
n=-\frac{R}{B} \frac{\partial B}{\partial R} \frac{1}{1+\dot{\phi}}-\frac{1}{B} \frac{\partial B}{\partial \theta} \frac{\tan (\phi)}{1+\dot{\phi}}
$$

Now, the median plane magnetic field can be written in cylindrical coordinates in the general form:

$$
B(R, \theta)=B_{m}(R) F(R, \theta),
$$

where $B_{m}(R)$ is the average magnetic field at a given radius which is $R$-dependent and $F(R, \theta)$ is the flutter function describing the azimuthal field variations along a fixed radius and satisfying

$$
\frac{1}{2 \pi} \int_{0}^{2 \pi} F(R, \theta) d \theta=1 .
$$

Taking the partial derivatives of the field in cylindrical coordinates yields

$$
\begin{gathered}
\frac{1}{B} \frac{\partial B}{\partial R}=\frac{1}{B_{m}(R)} \frac{\partial B_{m}}{\partial R}+\frac{1}{F(R, \theta)} \frac{\partial F(R, \theta)}{\partial R}, \\
\frac{1}{B} \frac{\partial B}{\partial \theta}=\frac{1}{F(R, \theta)} \frac{\partial F(R, \theta)}{\partial \theta} .
\end{gathered}
$$

Finally, the field index can be expressed as follows:

$$
n=-\frac{R}{B_{m}} \frac{\partial B_{m}}{\partial R} \frac{1}{1+\dot{\phi}}-\frac{R}{F} \frac{\partial F}{\partial R} \frac{1}{1+\dot{\phi}}-\frac{1}{F} \frac{\partial F}{\partial \theta} \frac{\tan (\phi)}{1+\dot{\phi}},
$$

and the character of the betatron oscillations is determined by Eqs. (25), which transform into

$$
\begin{aligned}
q_{x}(\theta)= & (1+\dot{\phi})^{2}+(1+\dot{\phi}) \frac{R}{B_{m}} \frac{\partial B_{m}}{\partial R} \\
& +(1+\dot{\phi}) \frac{R}{F} \frac{\partial F}{\partial R}+(1+\dot{\phi}) \tan (\phi) \frac{1}{F} \frac{\partial F}{\partial \theta}, \\
q_{y}(\theta)= & -(1+\dot{\phi}) \frac{R}{B_{m}} \frac{\partial B_{m}}{\partial R}-(1+\dot{\phi}) \frac{R}{F} \frac{\partial F}{\partial R} \\
& -(1+\dot{\phi}) \tan (\phi) \frac{1}{F} \frac{\partial F}{\partial \theta}, \\
f(\theta)= & \frac{\ddot{\phi}}{2} \tan (\phi)+\frac{\dot{\phi}^{2}-1}{4} \tan ^{2}(\phi)+\frac{\dot{\phi}}{2}(\dot{\phi}-1) .
\end{aligned}
$$

Note the analogy between Eq. (36) and Eq. (5.12) in Symon's paper [1], where all the derivations are based on generalized azimuthal coordinates: Both equations are exact in the sense that they describe the linear motion of the particles around the median plane closed orbit in any shape of magnetic field writing in cylindrical coordinates in the form given by Eq. (30). The main assumption in both derivations is that the magnetic field is perfectly symmetric with respect to the median plane. The first term in Eq. (36) represents the defocusing due to the average field index of the magnets. The spiral focusing comes predominantly from the second term. The third term is usually small except near the edge of the magnet; hence, it accounts for the edge focusing effect (often called Thomas focusing). Finally, the additional term in Eq. (35) comes from the horizontal restoring force.

Note, in addition, that the flutter function $F(R, \theta)$ can vanish in the straight sections, thus yielding a singularity in the above equations. However, it can be observed that the sign of the curvature function is related to the sign of the flutter function. Therefore, we shall seek a relationship between these two quantities. Before proceeding any further, we will assume that the flutter function does not exhibit any radial dependence. In other words, the condition of a radial sector FFA is fulfilled whereby $\partial F / \partial R=0$. However, the latter may not hold for nonscaling machines where the magnet is not sector shaped $[14,15]$. This will be discussed in a later publication.

\section{Flutter function}

First, let us compute the average magnetic field over a closed orbit $\mathcal{R}$ corresponding to a particle momentum $p$. From Eq. (14), one obtains 


$$
\langle B\rangle_{c o}=\frac{1}{2 \pi \mathcal{R}} \int_{0}^{2 \pi \mathcal{R}} \frac{p}{q} \frac{d s}{\rho(s)}=\frac{p}{q \mathcal{R}}
$$

where \langle\rangle$_{c o}$ refers to the average taken over the curvilinear abscissa. It results that

$$
B \rho=\frac{p}{q}=\langle B\rangle_{c o} \mathcal{R}
$$

For the case of a radial sector FFA, the flutter function is independent of the radius, and the expression of the magnetic field seen by the particle is written as a function of the azimuthal angle:

$$
B[R(\theta), \theta]=B_{m}[R(\theta)] F(\theta) .
$$

Now, equating the expression of the magnetic rigidity in both representations and making use of Eqs. (4), (11), and (39), we finally obtain

$$
\begin{aligned}
F(\theta) & =\frac{\langle B\rangle_{c o}}{B_{m}[R(\theta)]} \frac{\mathcal{R}}{R(\theta)}(1+\dot{\phi}) \cos (\phi) \\
& =\frac{\langle B\rangle_{c o}}{B_{m}[R(\theta)]} \frac{d \theta}{d \vartheta}(1+\dot{\phi}) .
\end{aligned}
$$

Thus, when assuming that the closed orbit does not depart much from a fixed radius orbit, i.e., $\phi \ll 1$, which is generally the case for fixed field accelerators, we can write as a first-order approximation

$$
\dot{\phi} \approx F(\theta)-1 .
$$

Nevertheless, the above approximation becomes less valid when the azimuthal variations as well as the radial increase of the field become large. For instance, for the KURNS FFA, the above formula is tested at injection energy where

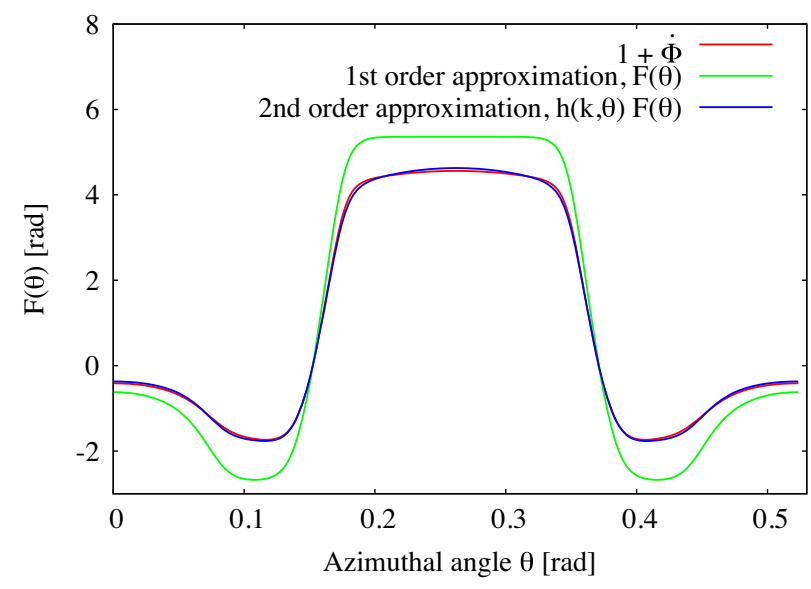

FIG. 4. Comparison of the tracking results (red curve) of the closed orbit scalloping at the KURNS FFA injection energy with its first- and second-order approximate expressions (green and blue curves, respectively). the orbit scalloping $\Delta R /\langle R\rangle$ is about $2 \%$, and we can observe in Fig. 4 a non-negligible difference between the two expressions. Thus, higher-order approximations are established (see the Appendix A) which are more accurate to explain the azimuthal variations of the scalloping angle as illustrated in Fig. 4.

\section{NUMBER OF BETATRON OSCILLATIONS FROM THE SECOND-ORDER DIFFERENTIAL EQUATION}

As was established by Teng [16], if we define $K_{x, y}(\theta)$ as the forcing term of the Hill's equation $\left[K_{x, y}(\theta)=q_{x, y}(\theta)+\right.$ $f(\theta)$ ], then the number of betatron oscillations can be approximated by

$$
\nu_{i}^{2} \approx\left\langle K_{i}\right\rangle+\left\langle\widetilde{K}_{i}^{2}\right\rangle+3\left\langle K_{i}\right\rangle\left\langle{\widetilde{K_{i}}}_{i}^{2}\right\rangle+\left\langle K_{i} \widetilde{\widetilde{K}}_{i}^{2}\right\rangle,
$$

where the subscript $i$ stands for $x$ or $y$, the symbol \langle\rangle represents the average taken over the azimuthal coordinate, and the tilde is the integrating operator defined by

$$
\begin{aligned}
& \tilde{g}(\theta)=\int[g(\theta)-\langle g\rangle] d \theta, \\
& \tilde{\tilde{g}}(\theta)=\int[\tilde{g}(\theta)-\langle\tilde{g}\rangle] d \theta .
\end{aligned}
$$

Such an approximation is generally referred to in the literature as the smooth approximation $[1,16]$ : Symon relied on such an approximation (limited to the first two terms) to derive the often-quoted formula for the tunes in FFA [see Eq. (1)].

For each closed orbit, there is a different set of linearized equations for the betatron oscillations, i.e., different pairs $\left[K_{x}(\theta), K_{y}(\theta)\right]$, so that the betatron wave numbers are susceptible to change with the energy. Our analysis is thus restricted to the fixed energy closed orbits, which is the commonly used approach to study the stability of the particle trajectories, since the acceleration process is adiabatic in scaling FFA.

For a radial sector FFA, when the closed orbit scalloping and the field gradients are small, i.e., $F(\theta) \sim 1$ and $k \lesssim 1$, the alternating gradient (AG) effect can be neglected. In other words, the tunes can be well approximated by the first-order term in Eq. (43), which is the average of the forcing term of the second-order linear differential equation (25): This accounts for the focusing (or defocusing) effect due to the mean restoring forces. Nevertheless, when the $k$ value increases substantially, such an approximation no longer holds, and it becomes important to account for the higher-order terms which are due to the AG forces that produce a substantial scalloping of the orbits alongside a stronger focusing. This is accounted for by the tilde functions, which represent the fluctuations about the mean value of the applied forces on the beam. 
In what follows, the different terms of the Hill's equation expressed in cylindrical coordinates will be discussed, and the emphasis made on their contribution to the number of betatron oscillations. Several formula will be established and their limit of validity discussed. To conclude, we will make a comparison between the tracking results and the analytical estimates for various values of the $F D$ ratio and the average field index of the magnets.

\section{A. Focusing due to the average field index}

If the averaged field (over the azimuth) increases with the radius, this will yield an overall focusing (respectively, defocusing) force in the horizontal (respectively, vertical) plane. Such a contribution to $q_{x}(\theta)$, denoted by the superscript ind, is defined by

$$
q_{x}^{\text {ind }}(\theta)=(1+\dot{\phi}) \frac{R}{B_{m}} \frac{\partial B_{m}}{\partial R}=(1+\dot{\phi}) k .
$$

Next, two concepts will be discussed based on the expression of the average magnetic field.

\section{Cyclotron}

In order to keep the isochronism in a cyclotron, the average magnetic field strength changes according to the law

$$
B_{m}(R)=B_{0} \gamma(R)=\frac{B_{0}}{\sqrt{1-\left(\frac{R}{R_{\infty}}\right)^{2}}},
$$

where $R_{\infty}$ is a constant. It results that

$$
\left\langle q_{x}^{\text {ind }}\right\rangle=k=\gamma^{2}-1,
$$

which shows that, in general, the average field index contributes weakly to the horizontal focusing in cyclotrons. Such a weak contribution is advantageous in the vertical plane, since it can be overcome by means of Thomas and/or spiral focusing.

\section{Scaling FFA}

For a perfect scaling FFA, $k$ is constant everywhere in the ring. It results that

$$
\left\langle q_{x}^{\text {ind }}\right\rangle=k=\text { const. }
$$

Since the $k$ value can be large, $k=7.6$ for the KURNS scaling FFA, it is obvious that the higher-order terms in Eq. (43) which account for the AG forces can no longer be neglected.

For instance, for a machine like the KURNS $150 \mathrm{MeV}$ FFA, we can estimate the contribution of the AG due to the field index to be

$$
\begin{aligned}
& \left\langle{\widetilde{q_{x}^{\text {ind }}}}^{2}\right\rangle=k^{2}\left\langle\phi^{2}\right\rangle \\
& \approx 7.6^{2}\left\langle[0.33 * \sin (12 \theta)]^{2}\right\rangle=3.14,
\end{aligned}
$$

which is about $2 / 5$ of the focusing due to the average field index. This is non-negligible and can place the tunes in the second stability region of Hill's equation, hence the concept of scaling FFA with a small orbit excursion [17]. However, this will be discussed more in detail later on in this paper where the AG focusing is evaluated.

\section{B. Thomas focusing}

From the previous analysis, the scalloping angle $\phi$ of the closed orbit can be accurately calculated using the second (or higher) order approximation established in Appendix A and written in the following general way:

$$
\dot{\phi}=h(k, \theta) F(\theta)-1
$$

[where $h(k, \theta)=1$ if we relied on the first-order approximation instead]. It results that the contribution of the Thomas focusing to the vertical tune can be simplified to

$$
\begin{aligned}
q_{y}^{\mathrm{th}}(\theta) & =-(1+\dot{\phi}) \tan (\phi) \frac{1}{F} \frac{\partial F}{\partial \theta} \\
& =-\tan (\phi) \ddot{\phi}+\frac{\partial h}{\partial \theta} \tan (\phi) F,
\end{aligned}
$$

thus removing its singularity.

By making use of Eq. (A4), we finally obtain

$$
q_{y}^{\text {th }}(\theta)=-\tan (\phi) \ddot{\phi}-(k+1) \tan (\phi)(1+\dot{\phi}) \tan (\tilde{F}) .
$$

In general, though, the right-hand-side term in the previous equation can be neglected in comparison with the left-handside term as long as $k \phi^{\max } / N \ll 1$. This is valid for all machines where the $k$ value is not exceedingly large, i.e., $k \sim 100$, which is not the focus of our analysis in the present paper.

By means of an integration by parts, the leading term of the Thomas focusing can be calculated:

$$
\left\langle q_{y}^{\text {th }}\right\rangle \approx\langle-\phi \ddot{\phi}\rangle=\left\langle\dot{\phi}^{2}\right\rangle=\left\langle[h(k, \theta) F(\theta)-1]^{2}\right\rangle .
$$

At this point, it is important to recall the contribution of the Thomas focusing to the vertical tune, which is usually quoted in the literature in several equivalent ways [1,18-22]:

$$
\begin{aligned}
\mathcal{F}^{2} & =\frac{\left\langle F^{2}\right\rangle-\langle F\rangle^{2}}{\langle F\rangle^{2}}=\frac{\left\langle B^{2}\right\rangle-\langle B\rangle^{2}}{\langle B\rangle^{2}}=\frac{f^{2}}{2} \\
& =\left\langle[F(\theta)-1]^{2}\right\rangle,
\end{aligned}
$$

where $\mathcal{F}^{2}$ is generally referred to as the magnetic flutter. The latter represents the fractional mean square azimuthal 
deviation of the field at a fixed radius. This term is large when the field is changing rapidly along the closed orbit. However, such a result is an approximation that is based on the assumption that the field changes slowly, both radially and azimuthally, and does not account for the variation of the orbit scalloping with the $k$ value. As is clearly stated in Ref. [22], "the most important of all these simplifications is the assumption of a small azimuthal variation of the field strength." To further illustrate this, both approximations [Eqs. (51) and (52)] are compared with the tracking results for various lattices where $k$ is varied while maintaining the flutter function $F(\theta)$ unchanged, and one can see in Fig. 5 that the second-order approximation reduces the error of the first-order estimate to below 5\%. In conclusion, the first-order approximation based on the expression (52) should be avoided, since it introduces an erroneous concept that the edge focusing is independent of the $k$ value of the magnet.

In general, Thomas focusing is dominated by the edge focusing effect and acts in a way that the resulting effect is a net restoring force in the vertical plane. It was Thomas in his seminal paper of 1938 [23] who showed for the first time that using an azimuthally varying field producing a scalloped particle orbit allows one to overcome the defocusing effect that would set the maximum energy of cyclotrons to $20 \mathrm{MeV}$ as claimed by Bethe and Rose [24].

Thomas's insight was to realize that the interaction between the radial component of the momentum and the azimuthal component of the magnetic field yields a vertical restoring force:

$$
\text { Force }_{y}=-q v_{R} B_{\theta}=-q \frac{d R}{d \theta} \frac{d \theta}{d t} B_{\theta}
$$

where $B_{\theta}$ is the azimuthal component of the magnetic field which is written for small $z$ [23]

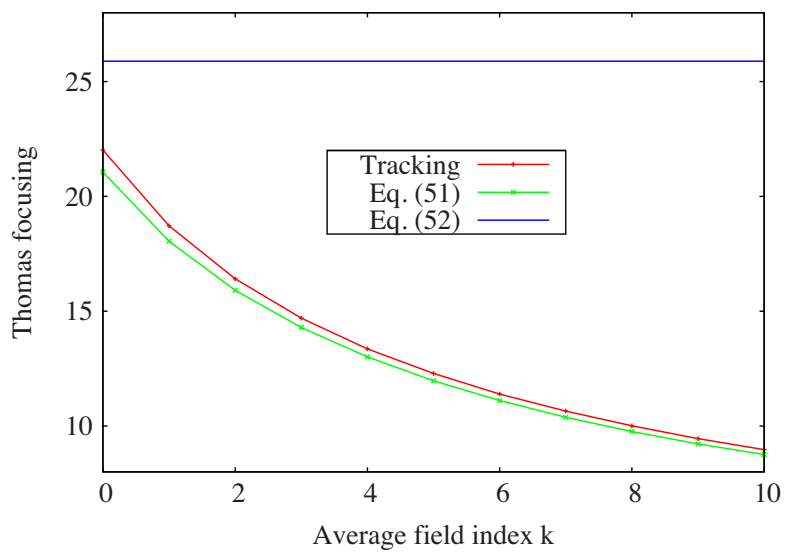

FIG. 5. Comparison of the tracking results for the Thomas focusing term $\left\langle q_{y}^{\text {th }}\right\rangle$ with the analytical estimates based on the first- and second-order approximations, Eqs. (52) and (51), respectively.

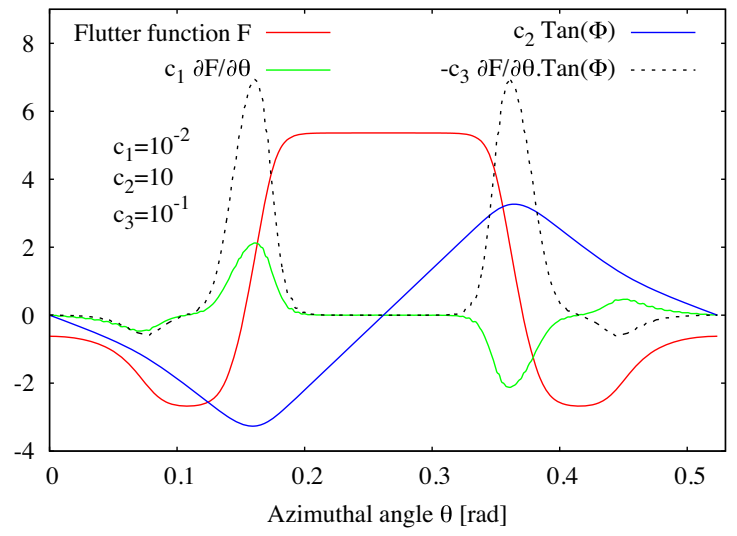

FIG. 6. Plot of the different terms contributing to the Thomas focusing at the injection energy of the KURNS scaling FFA: The orbit scalloping is shown via the angle $\phi$ (in blue), while the contribution of the field index is shown in green (partial derivative of the flutter function $F$ ). Their product is shown in dashed lines which account for the vertical focusing.

$$
B_{\theta}=\frac{z}{R} \frac{\partial B}{\partial \theta}+O\left(z^{3}\right) .
$$

Injecting the latter into the expression (53) and making use of Eq. (2) yields

$$
\text { Force }_{y}=q \frac{d \theta}{d t} \tan (\phi) \frac{\partial B}{\partial \theta} z .
$$

Such a force is usually directed toward the midplane, and, therefore, it is restoring in the vertical plane. Its average contribution to the vertical tune is approximately given by Eq. (51). This is further summarized in Fig. 6, displaying the tracking results from the KURNS $150 \mathrm{MeV}$ FFA. In some cases, it is asserted that the Thomas focusing was a special case of the general theory of the AG focusing. However, such a misconception is clarified in Ref. [25], and we make the distinction clear in the present paper.

\section{Horizontal restoring force}

Larger scalloping of the orbit is due to larger oscillations of the flutter function $F$ which can substantially increase the horizontal restoring force:

$$
\left\langle q_{x}^{\mathrm{res}}\right\rangle=\frac{1}{2 \pi} \int_{0}^{2 \pi}(1+\dot{\phi})^{2} d \theta=1+\left\langle\dot{\phi}^{2}\right\rangle .
$$

It results that, for the horizontal tune, the horizontal restoring force and the Thomas defocusing term compensate each other in such a way that

$$
\left\langle q_{x}^{\mathrm{res}}\right\rangle-\left\langle q_{y}^{\mathrm{th}}\right\rangle \approx 1
$$

In addition, stronger vertical focusing can be achieved if the magnet boundaries are deformed from radial poles to 
spiral-shaped poles. However, in the present paper, the focus of our analysis will be only on radial sector machines so that such a contribution is disregarded.

In the next subsection, we focus the analysis on obtaining simplified approximate expressions for the tunes in order to better understand when the alternating gradient focusing plays an important role in FFA.

Clearly, an exact solution of the problem can be obtained by relying on the Hill's equation of motion in cylindrical coordinates for which the scalloping angle is precisely solved as shown earlier.

\section{Alternating gradient focusing}

In summary, the expression of $K_{x}$ for a radial sector scaling FFA is written

$$
\begin{aligned}
K_{x}(\theta) & =q_{x}^{\mathrm{res}}(\theta)+q_{x}^{\text {ind }}(\theta)-q_{y}^{\mathrm{th}}(\theta)+f(\theta) \\
& \approx(1+\dot{\phi})^{2}+(1+\dot{\phi}) k+\frac{3}{2} \phi \ddot{\phi}+\frac{\dot{\phi}^{2}}{2}-\frac{\dot{\phi}}{2},
\end{aligned}
$$

where one neglected a small term involving $f(\theta)$ so that $\left\langle K_{x}\right\rangle \approx k+1$ and

$$
\begin{aligned}
\widetilde{K_{x}}(\theta) & =\int_{0}^{\theta}\left[K_{x}(u)-\left\langle K_{x}\right\rangle\right] d u \\
& \approx\left[k+\frac{3}{2}(1+\dot{\phi})\right] \phi ; \quad \phi(0)=0,
\end{aligned}
$$

where the reference azimuth is chosen such as $\phi(0)=0$. This is necessarily the case if $\theta=0$ is a point of symmetry of the orbit, i.e., $F(-\theta)=F(\theta)$. Similarly, we can compute the contribution of the AG to the vertical focusing:

$$
\begin{aligned}
K_{y}(\theta) & =-q_{x}^{\text {ind }}(\theta)+q_{y}^{\text {th }}(\theta)+f(\theta) \\
& \approx-(1+\dot{\phi}) k-\frac{\phi \ddot{\phi}}{2}+\frac{\dot{\phi}^{2}}{2}-\frac{\dot{\phi}}{2},
\end{aligned}
$$

which yields after integration $\left\langle K_{y}\right\rangle \approx-k+\left\langle\dot{\phi}^{2}\right\rangle$ and

$$
\begin{aligned}
\widetilde{K_{y}}(\theta) & =\int_{0}^{\theta}\left[K_{y}(u)-\left\langle K_{y}\right\rangle\right] d u \\
& \approx-\left[k+\frac{1}{2}(1+\dot{\phi})\right] \phi+\int_{0}^{\theta}\left[\dot{\phi}^{2}-\left\langle\dot{\phi}^{2}\right\rangle\right] d u .
\end{aligned}
$$

To facilitate further discussion, we make the following considerations: Let us assume that $F(\theta)$ is an even function in $\theta$ as illustrated in Fig. 4. It results that its integral and, therefore, the scalloping angle $\phi$ are odd functions in $\theta$. Thus, $\phi$ is written in the form

$$
\phi(\theta)=\sum_{j=1}^{\infty} \phi_{j} \sin (j N \theta),
$$

where $\phi_{j}$ are the coefficients of the Fourier series and $N$ is the total number of sectors in the ring. To simplify the analysis, we shall keep only the first two terms in this series:

$$
\phi(\theta)=\phi_{1} \sin (N \theta)+\phi_{2} \sin (2 N \theta) .
$$

Now, making use of Eqs. (58) and (59) yields

$$
\begin{aligned}
\left\langle\widetilde{K}_{x}^{2}\right\rangle= & \left(k+\frac{3}{2}\right)^{2} \frac{\phi_{1}{ }^{2}+\phi_{2}{ }^{2}}{2}+\frac{9 N^{2}}{32}\left(\phi_{1}{ }^{4}+4 \phi_{2}{ }^{4}\right) \\
& +\frac{45}{16} N^{2} \phi_{1}{ }^{2} \phi_{2}{ }^{2}
\end{aligned}
$$

and

$$
\begin{aligned}
\left\langle\widetilde{K}_{y}{ }^{2}\right\rangle= & \left(k+\frac{1}{2}\right)^{2} \frac{\phi_{1}{ }^{2}+\phi_{2}{ }^{2}}{2}+\frac{365}{144} N^{2} \phi_{1}{ }^{2} \phi_{2}{ }^{2} \\
& -\frac{9}{4}\left(k+\frac{1}{2}\right) N \phi_{1}{ }^{2} \phi_{2} .
\end{aligned}
$$

If $\phi_{2}=0$, then an approximate expression of the tunes is written as follows:

$$
\begin{gathered}
\nu_{x} \approx\left[k+1+\left(k+\frac{3}{2}\right)^{2}\left\langle\phi^{2}\right\rangle+\frac{9}{8} N^{2}\left\langle\phi^{2}\right\rangle^{2}\right]^{1 / 2}, \\
\nu_{y} \approx\left[-k+\left\langle\dot{\phi}^{2}\right\rangle+\left(k+\frac{1}{2}\right)^{2}\left\langle\phi^{2}\right\rangle\right]^{1 / 2},
\end{gathered}
$$

where we assumed that most of the focusing comes predominantly from the first two terms in the tune expression (43) and where the scalloping angle $\phi$ is obtained from the second (or higher) order approximation, $\phi \approx h(k, \widetilde{\theta) F}(\theta)$.

The above expressions of the tunes show that the AG effect is generally more important in the horizontal plane than in the vertical one. The latter is particularly sensitive to the shape of the closed orbit. In addition, the contribution of the AG to the transverse focusing increases with the number of sectors.

Last but not least, achieving a constant $k$ value is not a sufficient condition in order to obtain a fixed tune machine: If the flutter function $F(\theta)$ changes from low energies to high energies, this will necessarily introduce a change in the scalloping angle of the closed orbit, $\phi(k, \theta) \approx$ $h(k, \widetilde{\theta) F}(\theta)$ and, therefore, the tunes as well.

Now, as a verification example, the second-order analytical approximation of the scalloping angle $\phi$ at the injection energy of the KURNS $150 \mathrm{MeV}$ FFA where $k=7.6$ yields $\left\langle\phi^{2}\right\rangle=0.036$ and $\left\langle\dot{\phi}^{2}\right\rangle=6.76$ (while the first order yields $\mathcal{F}^{2}=10.74$ ). Injecting the latter into Eqs. (64) and (65) yields 
TABLE I. Comparison of the ZGOUBI tracking results with the approximate formula for various values of the average field index $k$. The FD ratio is fixed such that $\mathcal{F}^{2}=25.89$.

\begin{tabular}{|c|c|c|c|c|c|c|c|}
\hline \multirow[b]{2}{*}{ Field index $k$} & \multicolumn{2}{|c|}{ Tracking } & \multicolumn{2}{|c|}{ First order } & \multicolumn{2}{|c|}{ Third order } & \multirow[b]{2}{*}{ Edge focusing $\left\langle q_{y}^{\text {th }}\right\rangle$} \\
\hline & $\nu_{x}^{2}$ & $\nu_{y}^{2}$ & $\left\langle K_{x}\right\rangle$ & $\left\langle K_{y}\right\rangle$ & $\left\langle K_{x}\right\rangle+\left\langle\widetilde{K}_{x}^{2}\right\rangle+\cdots$ & $\left\langle K_{y}\right\rangle+\left\langle\widetilde{K}_{y}^{2}\right\rangle+\cdots$ & \\
\hline 0 & 1.41 & 22.89 & $(-1.30)$ & 21.73 & 1.14 & 23.04 & 22.03 \\
\hline 1 & 2.98 & 17.75 & 0.40 & 17.50 & 2.88 & 17.82 & 18.72 \\
\hline 2 & 4.69 & 14.35 & 1.84 & 14.24 & 4.68 & 14.34 & 16.42 \\
\hline 3 & 6.58 & 11.80 & 3.14 & 11.55 & 6.63 & 11.79 & 14.70 \\
\hline 4 & 8.64 & 9.76 & 4.37 & 9.24 & 8.76 & 9.77 & 13.36 \\
\hline 5 & 10.95 & 8.06 & 5.54 & 7.17 & 11.08 & 8.05 & 12.28 \\
\hline 6 & 13.58 & 6.59 & 6.68 & 5.29 & 13.62 & 6.50 & 11.39 \\
\hline 7 & 16.68 & 5.30 & 7.80 & 3.55 & 16.37 & 5.04 & 10.64 \\
\hline 8 & 20.58 & 4.14 & 8.89 & 1.92 & 19.35 & 3.60 & 10.00 \\
\hline 9 & 24.40 & 3.08 & 9.97 & 0.37 & 22.53 & 2.12 & 9.44 \\
\hline 10 & 28.87 & 2.18 & 11.04 & $(-1.11)$ & 25.95 & 0.59 & 8.96 \\
\hline
\end{tabular}

$$
\begin{aligned}
& \nu_{x} \approx[8.6+2.98+0.2]^{1 / 2}=3.43, \\
& \nu_{y} \approx[-7.6+6.76+2.36]^{1 / 2}=1.23,
\end{aligned}
$$

which is consistent with the tracking simulation results relying on the simulated field map using the OPERA-3DTOSCA package [26] as shown in Fig. 1. In addition, it is clear that the AG effect is negligible for radial sector fixed field machines where the $k$ value is not important such as cyclotron accelerators or for machines where the orbit scalloping is small, i.e., $F(\theta) \approx 1$. When the $k$ value is nonnegligible as well as the orbit scalloping, which is the focus of our analysis, then the net focusing is due to a mixing between the average forces applied on the beam and the fluctuations around the mean values of these forces. It is important to point out that the approximate expressions shown above cannot be more accurate than the tracking results. However, they are sufficiently accurate to serve as an important guideline for the design of the magnet and the verification of the convergence of the tracking simulations.

\section{E. Benchmarking the analytical formula with tracking simulations}

In summary, the number of betatron oscillations for a radial sector scaling FFA can be determined from the expressions of $K_{x}$ and $K_{y}$ :

$$
\begin{aligned}
& K_{x}(\theta)=q_{x}^{\mathrm{res}}(\theta)+q_{x}^{\mathrm{ind}}(\theta)-q_{y}^{\mathrm{th}}(\theta)+f(\theta), \\
& K_{y}(\theta)=-q_{x}^{\text {ind }}(\theta)+q_{y}^{\mathrm{th}}(\theta)+f(\theta),
\end{aligned}
$$

where $f(\theta)$ is given by Eq. (25) such that its first-order contribution is a small defocusing effect in both planes:

$$
\langle f\rangle=-\frac{\left\langle p^{2}\right\rangle}{4}=-\frac{1}{4}\left\langle(1-\dot{\phi})^{2} \tan ^{2}(\phi)\right\rangle,
$$

and the tunes are evaluated by means of Eq. (43). The scalloping angle of the closed orbits is obtained by solving the coupled equations (A1) and (A2) using the method of successive approximations discussed in Appendix A. The closed orbit results are in excellent agreement with the tracking simulation results [below $1 \mathrm{mrad}$ absolute error on $\phi(\theta)$ with the fifth-order approximation].

Table I shows a comparison of the tracking results of radial sector scaling FFA with the analytical expressions for different values of the average field index: As can be seen, when increasing the $k$ value, the vertical tune decreases. However, this is explained by two main contributions: an increasing average field index as well as a decreasing edge focusing. The latter is particularly sensitive to the shape of the equilibrium orbit, which depends on the average field index as well as the flutter function. In addition, it can be seen that the often-quoted formulas $\nu_{x}^{2} \approx k+1$ and $\nu_{y}^{2} \approx$ $-k+\mathcal{F}^{2}$ do predict the qualitative behavior. However, they fail to predict the quantitative (or monotonic behavior as will be shown later on in this paper): The first approximation, $\nu_{x}^{2} \approx k+1$, does underestimate the horizontal tunes, since it does not account for the $A G$ forces. However, the second one, $\nu_{y}^{2} \approx-k+\mathcal{F}^{2}$, does overestimate the vertical tunes, since it does not account for the reduced scalloping of the orbit which reduces substantially the edge focusing effect. In general, for small $k$ values, we can observe that the vertical tunes are well approximated by the first-order formula, $\left\langle K_{y}\right\rangle$, i.e., the average values of the transverse forces applied on the beam. This is the cyclotron regime for which the average field index does not depart much from $\gamma^{2}-1$. Nevertheless, the horizontal tunes are not well predicted by such an approximation. The reason is due to the $F D$ ratio, which is defined as the absolute value of the ratio between the minimum and maximum of the flutter function, i.e., $F D=|\min (F) / \max (F)|$. The latter is non-negligible in this example, $F D=0.69$, so that the AG forces dominate in the horizontal plane, hence the need to account for the additional focusing terms given by Eq. (43). This is further illustrated in Table II, where the discrepancy between the tracking and the first-order formula increases with an increasing $F D$ ratio. 
TABLE II. Comparison of the ZGOUBI tracking results with the approximate formula for various $F D$ ratios $(k=5)$.

\begin{tabular}{|c|c|c|c|c|c|c|c|}
\hline \multirow[b]{2}{*}{$F D$ ratio } & \multicolumn{2}{|c|}{ Tracking } & \multicolumn{2}{|c|}{ First order } & \multicolumn{2}{|c|}{ Third order } & \multirow[b]{2}{*}{ Edge focusing $\left\langle q_{y}^{\text {th }}\right\rangle$} \\
\hline & $\nu_{x}^{2}$ & $\nu_{y}^{2}$ & $\overline{\left\langle K_{x}\right\rangle}$ & $\overline{\left\langle K_{y}\right\rangle}$ & $\left\langle K_{x}\right\rangle+\left\langle\widetilde{K}_{x}^{2}\right\rangle+\cdots$ & $\left\langle K_{y}\right\rangle+\left\langle\widetilde{K}_{y}^{2}\right\rangle+\cdots$ & \\
\hline 0.30 & 7.58 & 0.0023 & 6.00 & -0.29 & 7.81 & -0.003 & 4.74 \\
\hline 0.43 & 8.30 & 1.88 & 5.94 & 1.48 & 8.57 & 1.85 & 6.52 \\
\hline 0.58 & 9.35 & 4.50 & 5.81 & 3.91 & 9.64 & 4.48 & 8.98 \\
\hline 0.72 & 10.95 & 8.06 & 5.54 & 7.17 & 11.08 & 8.05 & 12.28 \\
\hline 0.85 & 13.44 & 12.81 & 5.06 & 11.30 & 12.99 & 12.74 & 16.48 \\
\hline 1.00 & 17.62 & 19.11 & 4.31 & 15.72 & 14.86 & 17.70 & 21.00 \\
\hline
\end{tabular}

Nevertheless, it appears that the smooth approximation fails near the stability boundary, hence the need to extend the approximation to higher-order terms [16]. However, we will not pursue this here.

\section{BEAM STABILITY ANALYSIS}

Technically, it is impossible to make a field which corresponds exactly to the designed one. Therefore, it is important to understand the effect of small imperfections of the field on the beam dynamics.

In the following, we investigate the stability of the particle trajectories that can arise with different field errors. We use two different approaches to investigate the beam stability due to field errors: The first approach is based on the previously established analytical solution of the betatron wave numbers by means of a smooth approximation of the linearized equations of motion. The second model is the ZGOUBI [11] tracking model of the magnet, which is the most accurate one. The ZGOUBI model solves the nonlinear equation of motion using field maps or user-implemented analytical models. To conclude, we establish a comparison between the different results and comment on the outcome of this study.

\section{A. Field imperfections in scaling FFAs}

For an ideal radial sector scaling FFA, the magnetic field is written in cylindrical coordinates in the following way:

$$
B(R, \theta)=B_{0}\left(\frac{R}{R_{0}}\right)^{k} F(\theta) .
$$

Thus, a natural way to verify the validity of a calculated field map is to introduce a generalized definition of the average field index which accounts for the local imperfections of the field (radially and azimuthally):

$$
k(R, \theta)=\frac{R}{B} \frac{\partial B}{\partial R},
$$

where the field-free region is excluded from the definition of $k(R, \theta)$.

In order to avoid any confusion, it is important to remind the definition of the average field index introduced by Symon, which is given by

$$
k_{\text {symon }}=\frac{\mathcal{R}}{\langle B\rangle_{\text {co }}} \frac{d\langle B\rangle_{\mathrm{co}}}{d \mathcal{R}} .
$$

If Eq. (67) holds everywhere in the ring, then $k(R, \theta)=$ $k_{\text {symon }}$ is constant and both definitions are equivalent. Nevertheless, due to imperfections, we expect some variations of the above defined quantities as illustrated in Fig. 7.

\section{Field map derivative}

Using a TOSCA 2D median plane field map of the KURNS FFA which is simulated using OPERA finite element software, we calculated the field map derivative $k(R, \theta)$ of the main magnets, i.e., the focusing $F$ magnet and the defocusing $D$ one. The result is shown in Figs. 8 and 9 , where a non-negligible variation of this quantity is observed: For the $F$ magnet, the variations of $k$ are small, and the latter is close to its design value $k=7.6$. Nevertheless, for the $D$ magnet, the variations of $k$ are important. The main source of discrepancy is observed in the interaction region between the two magnets and seems to affect mainly the defocusing one in the neighborhood of the injection radius. Each sector in the ring is comprised of a $D F D$ triplet configuration which is symmetrical around

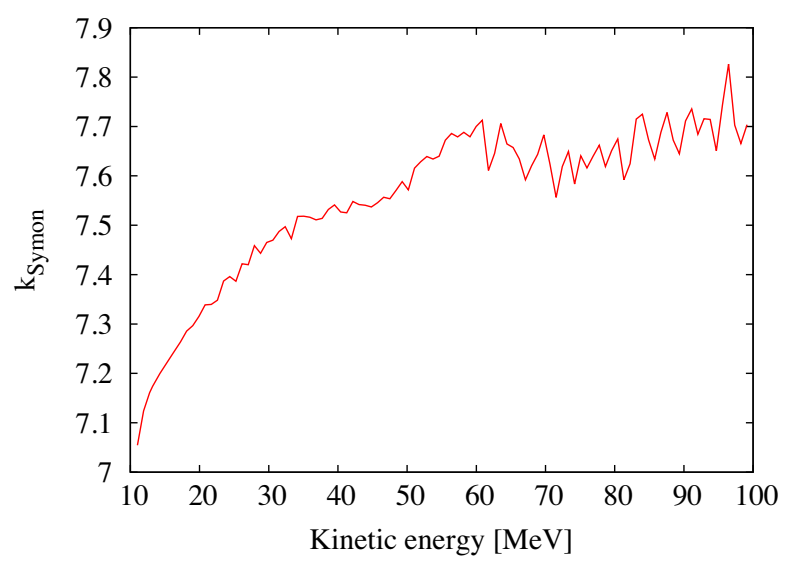

FIG. 7. Plot of the average field index $k_{\text {symon }}$ as a function of the kinetic energy for the KURNS $150 \mathrm{MeV}$ scaling FFA. Note the oscillatory behavior which is due to the granularity of the field map. 


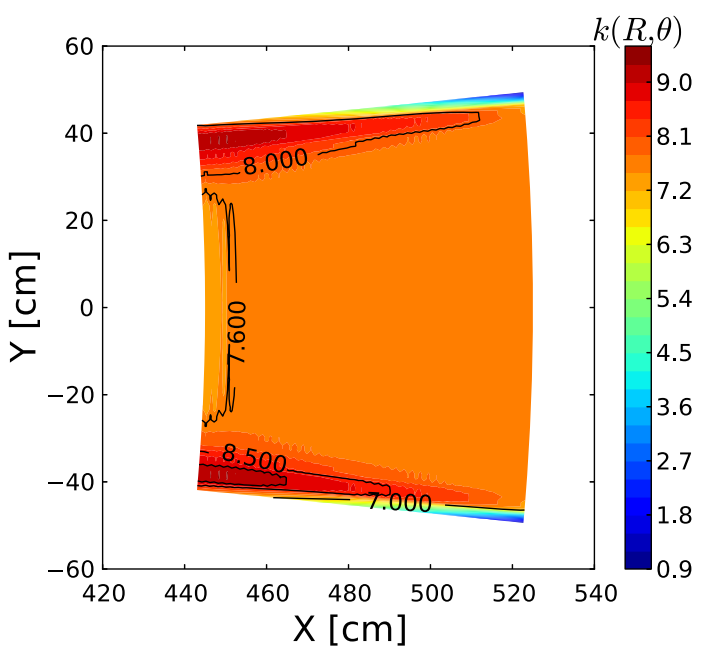

FIG. 8. Plot of the average field index map of the $F$ magnet. The central line $(Y=0)$ is a line of symmetry of the $D F D$ triplet.

the center of the $F$ magnet. Besides, a "return-yoke-free" design has been developed to ease the problem of variable energy extraction. This means that the flux generated by the $F$ poles return through the $D$ poles $[26,27]$. Nevertheless, this causes a non-negligible leakage field in the straight section and affects mainly the $D$ magnet. In order to have a better understanding of the impact of such a discrepancy, we can simplify the model by assigning an average field index to each magnet. The latter is calculated in the central region of each and evolves with the radius as shown in Fig. 10.

\section{B. Generalized model of imperfect scaling radial sector FFA}

In order to carry out parametric studies of the field defects in scaling FFAs, we assign an average field index to

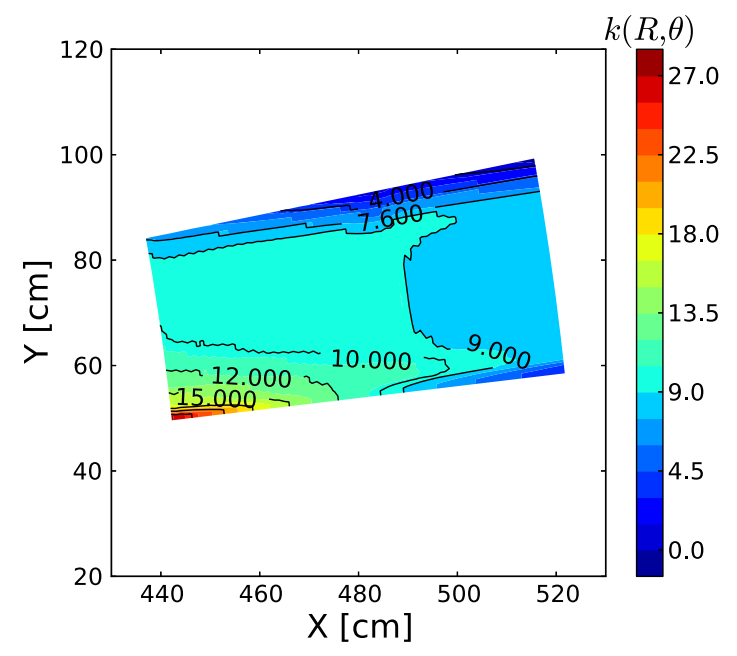

FIG. 9. Plot of the average field index map of the $D$ magnet. The lower part is in the neighborhood of the $F$ magnet, while the upper part is surrounded by the drift space separating the sectors.

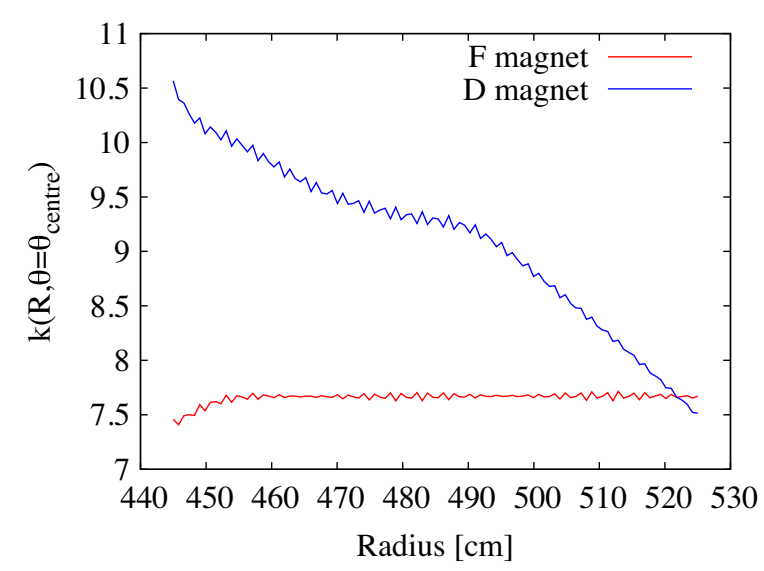

FIG. 10. $k(R, \theta)$ in the center of the $F$ and $D$ magnets.

each magnet that is not necessarily equal to the ideal one. Thus, we define

$$
k_{i}=\frac{R}{B_{i}} \frac{\partial B_{i}}{\partial R} ; \quad i=F, D
$$

where $B_{i}$ is the vertical component of the magnetic field in the median plane of the FFA magnet. Now, assuming that the $k$ values have no radial dependence (a complete derivation of the expression of the field when $k$ is $R$ dependent can be found in Appendix B), Eq. (70) can be integrated and the magnetic field expressed in cylindrical coordinates:

$B(R, \theta)=B_{F 0}\left(\frac{R}{R_{0}}\right)^{k_{F}} F_{F}(\theta)+a B_{D 0}\left(\frac{R}{R_{0}}\right)^{k_{D}} F_{D}(\theta)$,

where $F_{F}$ and $F_{D}$ are the fringe field factors (or flutter functions) that describe the azimuthal variation of the field in the $F$ and $D$ magnets, respectively, $B_{F 0}$ and $B_{D 0}$ are chosen such that $\left\langle F_{F}\right\rangle=-\left\langle F_{D}\right\rangle=1$, and $a$ is a scale factor that allows one to vary the $F D$ ratio of the magnet $(a \geq 0)$. It is important to note that the field is a separable function in radial and azimuthal coordinates, since, in our model, the fringe fields merge to zero between the magnets as can be seen in Fig. 11; thus, $F_{F}(\theta) F_{D}(\theta)=0$. Also, note that if $k_{F}=k_{D}$, the field is written in the standard form of a scaling FFA. The lattice considered for this study is a radial sector KURNS-like $D F D$ triplet [28]. It results that

$$
\begin{gathered}
\frac{1}{B} \frac{\partial B}{\partial \theta}=\frac{1}{F_{i}} \frac{\partial F_{i}}{\partial \theta}=\frac{1}{F} \frac{\partial F}{\partial \theta}, \\
\frac{1}{B} \frac{\partial B}{\partial R}=\frac{k_{i}(R)}{R},
\end{gathered}
$$

where $F(\theta)=F_{F}(\theta)+F_{D}(\theta)$. 


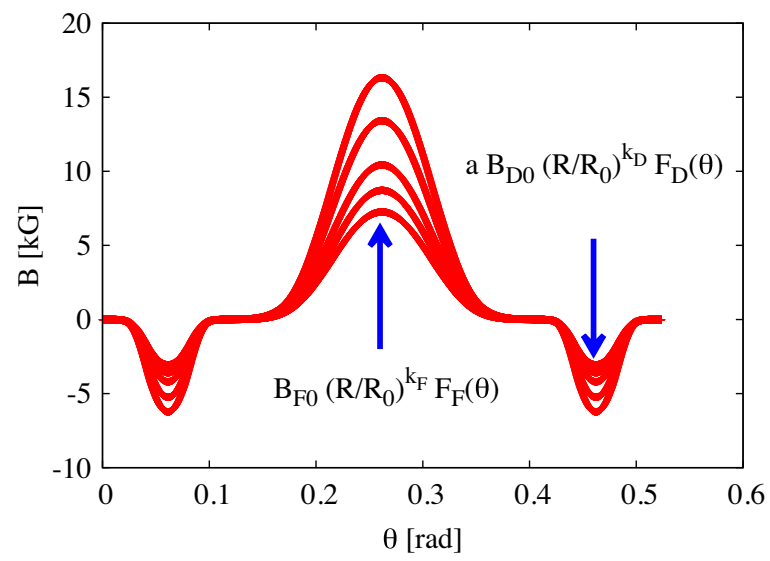

FIG. 11. Magnetic field along several closed orbits in a $D F D$ triplet.

\section{Monotonic behavior of the betatron wave numbers \\ 1. Conjecture}

In this section, we make the following conjecture regarding the impact of scaling imperfections on the monotonic behavior of the tunes.

In the presence of scaling imperfections, the number of betatron oscillations per turn increases (respectively, decreases) with the energy if $\kappa>0$ (respectively, $\kappa<0$ ) where $\kappa=k_{D}-k_{F}$. Besides, the variation of the tune squared is, to the first order, proportional to $|\kappa|$, i.e., $\Delta\left(\nu_{x, y}^{2}\right) \approx a_{x, y}|\kappa|$.

From what preceded, it was shown that the shape of the equilibrium orbit which is determined by the scalloping angle $\phi$ is the fundamental parameter that determines whether the closed orbits do or do not scale. In a nearly scaling FFA where the $k$ value of the $D$ magnet is larger than that of the $F$ magnet, this is to some extent equivalent to increasing the $F D$ ratio with the energy and thereby to increasing the scalloping of the closed orbits with the energy. The impact of increasing the scalloping of the orbits is, therefore, to increase the edge focusing effect as well as the AG focusing with the energy. Thus, by means of Eqs. (64) and (65), we shall expect that the tunes will increase monotonically with the energy of the particle if $\kappa>0$. The opposite effect occurs when $\kappa<0$.

For the second part of the conjecture, we shall evaluate the flutter function which is written in cylindrical coordinates as follows:

$$
F(R, \theta)=\frac{B(R, \theta)}{\langle B(R, \theta)\rangle}=\frac{F_{F}(\theta)+A\left(\frac{R}{R_{0}}\right)^{\kappa} F_{D}(\theta)}{1-A\left(\frac{R}{R_{0}}\right)^{\kappa}},
$$

where $0<A=a B_{D 0} / B_{F 0}<\left(R_{0} / R\right)^{\kappa}$. For small scaling imperfections, the flutter function evolves slowly with the radius. Thus, an approximate expression of $F$ for a given closed orbit of average radius $r$ is

$$
F(r, \theta)=\frac{F_{F}(\theta)+A\left(\frac{r}{R_{0}}\right)^{\kappa} F_{D}(\theta)}{1-A\left(\frac{r}{R_{0}}\right)^{\kappa}} .
$$

To simplify the analysis, we rely on the first-order approximation of the scalloping angle:

$$
\begin{aligned}
\left\langle\dot{\phi}^{2}\right\rangle & \approx\left\langle F(r, \theta)^{2}\right\rangle-1 \\
& =\frac{\left\langle F_{F}^{2}\right\rangle+A^{2}\left\langle F_{D}^{2}\right\rangle\left(\frac{r}{R_{0}}\right)^{2 \kappa}}{\left[1-A\left(\frac{r}{R_{0}}\right)^{\kappa}\right]^{2}}-1,
\end{aligned}
$$

which is an increasing (respectively, decreasing) function of $r$ if $\kappa>0$ (respectively, $\kappa<0$ ). Furthermore, the overall variation of the edge focusing can be estimated as follows:

$$
\begin{aligned}
\left|\left\langle\dot{\phi}^{2}\right\rangle^{\max }-\left\langle\dot{\phi}^{2}\right\rangle^{\min }\right| \approx \mid \frac{\left\langle F_{F}^{2}\right\rangle+A^{2}\left\langle F_{D}^{2}\right\rangle\left(\frac{r}{R_{0}}\right)^{2 \kappa}}{\left[1-A\left(\frac{r}{R_{0}}\right)^{\kappa}\right]^{2}} \\
-\frac{\left\langle F_{F}^{2}\right\rangle+A^{2}\left\langle F_{D}^{2}\right\rangle}{[1-A]^{2}} \mid \\
\approx \\
\approx \frac{2\left[A\left\langle F_{D}^{2}\right\rangle+\left\langle F_{F}^{2}\right\rangle\right]}{(1-A)^{3}} A \frac{\Delta r}{R_{0}}|\kappa| \propto|\kappa|,
\end{aligned}
$$

which partially establishes the conjecture above in the vertical plane. Similarly, the impact of the AG focusing on the tunes can be evaluated by calculating $\left\langle\phi^{2}\right\rangle$. The result will be in the same form.

Equation (78) shows that reducing the $F D$ ratio helps reduce the tune variations. This is expected, since, in our model, the $D$ magnet is the source of the field defect. Furthermore, increasing the alternation of the gradient increases the sensitivity of the tunes to the field imperfections via the second-order moments of the flutter functions, i.e., $\left\langle F_{D}^{2}\right\rangle$ and $\left\langle F_{F}^{2}\right\rangle$. In addition, it is shown that the effect of the scaling imperfections on the tune variations grows linearly with the radial excursion of the orbits in both horizontal and vertical planes. This shows the advantages of having an $F D F$ triplet configuration (rather than a $D F D$ one) with a much larger field index, since then the orbit excursion can be reduced by a factor of 5 or more [29], leading to much lower tune variations due to field errors of the type described above.

\section{Tracking simulations}

Now, we demonstrate that our findings with the previous analysis are reinforced by numerical simulations.

From the smooth approximation, it was found that the tune is sensitive to the average field index $k_{F}$ and $k_{D}$ of the $F$ and $D$ magnet, respectively. In other words, breaking the scaling law, although a major source of imperfection in scaling FFA, can also be utilized in order to control the tune path in FFA. In order to quantify the source of imperfection, 
we introduce two new quantities in the calculation: the average value of the tunes $\nu_{x}^{m}=\left\langle\nu_{x}\right\rangle$ and $\nu_{y}^{m}=\left\langle\nu_{y}\right\rangle$ over the range of energies, to quantify the average focusing strength of the applied forces on the beam, and the rms value of the tunes $\nu_{x}^{\mathrm{rms}}=\sigma_{\nu_{x}}$ and $\nu_{y}^{\mathrm{rms}}=\sigma_{\nu_{y}}$, to quantify the scaling imperfections in terms of tune variations. One could instead use the $|\max -\min |$ value of the tunes to account for the oscillations. However, the rms quantities have the merit to be average quantities and, thus, more appealing to use in order to obtain smooth variations of the described quantities.

\section{Benchmarking work}

Following the FFA workshop held at BNL [30], a simulation campaign was established to benchmark several simulation codes. The main objective is to provide reliable modeling tools for FFA type of accelerators and to better explain the results of the experiments at the KURNS $150 \mathrm{MeV}$ scaling FFA [7]. There exist several simulation codes for FFAs [31]. However, only a few were part of this benchmarking analysis so far. For instance, the CYCLOPS code, which is probably the best known tool for beam dynamics simulations in cyclotrons, can also be used for the simulation of FFA, yielding results in excellent agreement with the others [32]. Nevertheless, the aim of the present paper is not to compare codes.

The first benchmarking test was carried out for the calculation of the betatron tune as a function of the momentum and shows excellent agreement between the different codes (that were part of this campaign) as shown in Fig. 12. The setup of the benchmarking model as well as the details of the simulation can be found in Ref. [33].

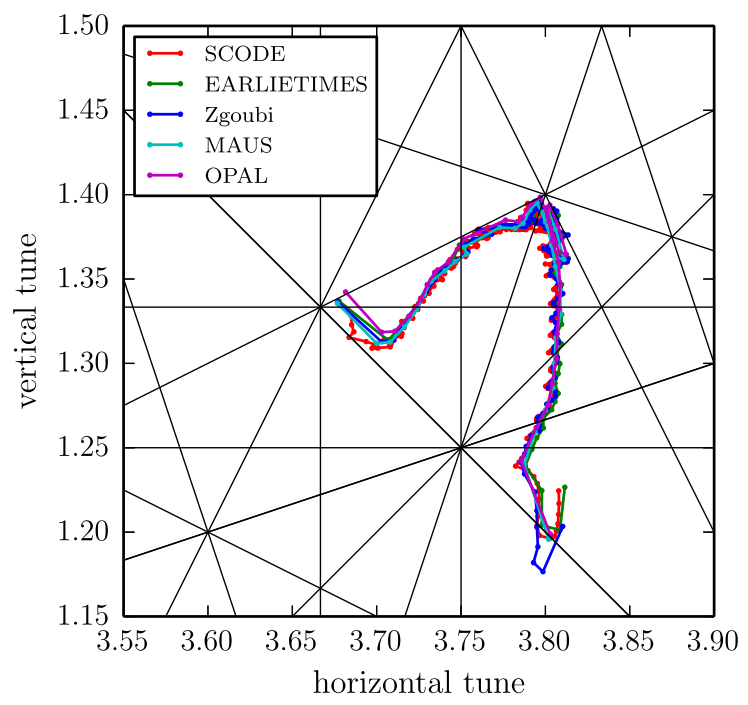

FIG. 12. Betatron tunes from 11 to $139 \mathrm{MeV}$ (left to right) calculated with several codes [33]. The ZGOUBI model is in good agreement with the others. The solid lines, in black, show the resonance lines up to the fourth order.

\section{ZGOUBI tracking model}

Based on the successful benchmarking test, we carry out parametric studies based on the ZGOUBI tracking code. The ZGOUBI code solves the nonlinear equation of motion using truncated Taylor expansions of the field and its derivatives up to the fifth order. Thus, it is more accurate than the linear approach. Given that the energy gain per turn is small in scaling FFAs (typically $2 \mathrm{keV}$ per turn in the KURNS machine), it can be reasonably assumed that the accelerated orbit trajectory for any given energy is quasi the same as the closed orbit trajectory. Thus, the procedure employed for the calculation of the betatron wave number is based on the closed orbits formalism described below.

First, a median plane field map is generated for a given $\left(a, k_{F}, k_{D}\right)$ as shown in Fig. 11. The field falloff at the end of the magnets is obtained by using an Enge-type fringe field model [34]. Extrapolation off the median plane is then achieved by means of Taylor series: For that, the median plane symmetry is assumed, and the Maxwell equations are accommodated. This yields results in excellent agreement with the 3D field map calculation.

Second, a search for a number of closed orbits between injection and extraction is performed using the built-in fitting routines in ZGOUBI. This quantity denoted NCO was chosen to be 30 in order to have good statistics and ensure the convergence of the average as well as the rms calculated quantities. A typical example of four closed orbits search is illustrated in Fig. 13.

Lastly, for each closed orbit, the betatron wave number is calculated in both planes.

Figure 14 shows the stability diagram obtained by varying the average field index of the magnets $(\kappa=0)$ as well as their $F D$ ratio and, therefore, the scale factor $a$ in

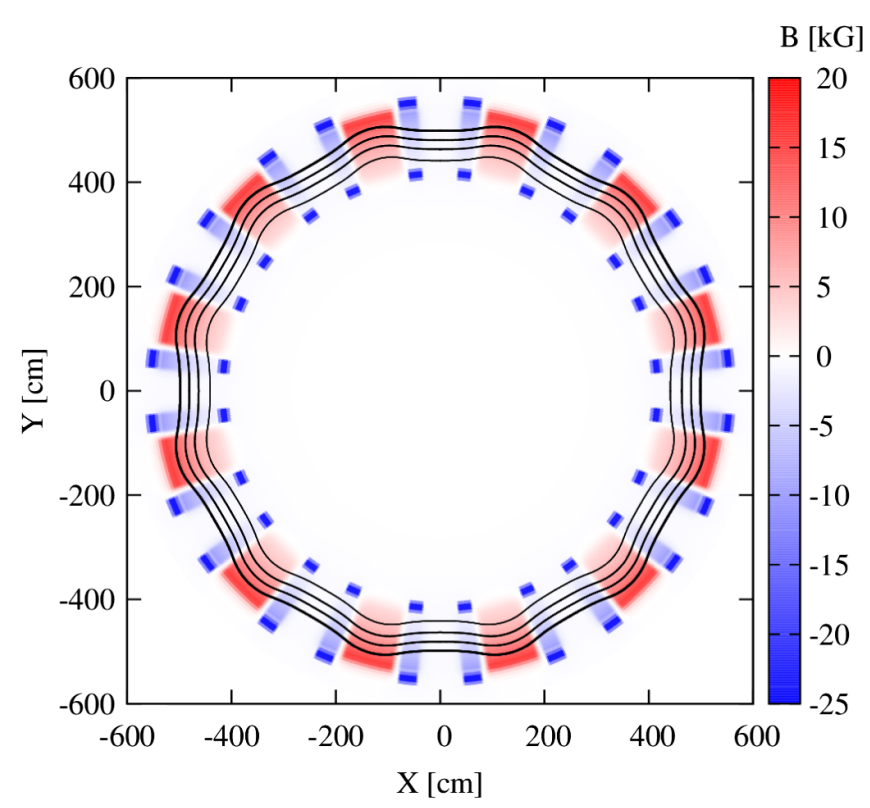

FIG. 13. Example of several closed orbits for a scaling FFA. 


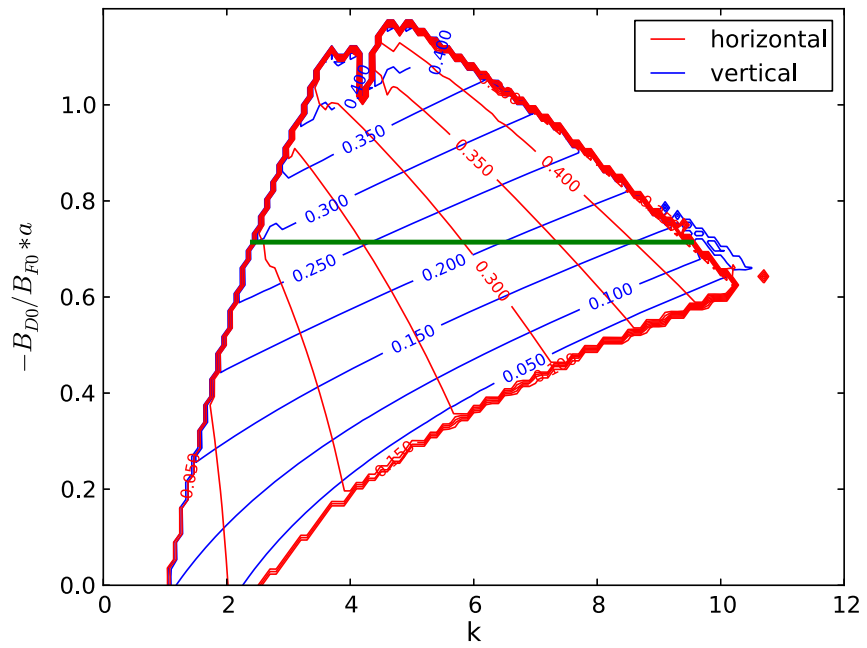

FIG. 14. Stability diagram as a function of the $F D$ ratio and the average field index $k$ : The green line shows the value of the $F D$ ratio that we choose for the study that follows. The number along with the red and blue lines are the horizontal and vertical cell tunes, respectively. Note that the region on the left side is limited by the physical size of the magnets, which is limited to $R_{\max }=10 \mathrm{~m}$.

Eq. (71). It can be seen that, on the top and bottom right, the stability limits are set by the horizontal and vertical cell tunes, respectively. On the left side, the physical size of the magnets (here, a radial excursion limited to $10 \mathrm{~m}$ is chosen) determines the boundary limits.

Now, we choose to focus our analysis on the average field index of the magnets. For that, we fix the $F D$ ratio. We choose $a=1$, which corresponds to the green line in Fig. 14. A scan on $k_{F}$ and $k_{D}$ provides the stability diagram of the DFD triplet in the transverse plane (see Fig. 15). Qualitatively, it shows that, in the case where $k_{F}=k_{D}=k$, the average cell tune exhibits the expected behavior predicted by the Symon formula, i.e., Eq. (1): Increasing $k$ increases the horizontal tune and decreases the vertical one. We can also observe that, for large $k$ values, the stability diagram shrinks; thus, any design imperfection will make the orbits quickly unstable. This is explained in the following way: On the right side of the stability diagram, i.e., when $\kappa<0$, the stability limit is set by the condition that $\nu_{y}^{2}$ is to remain positive (Floquet resonance), given that the tunes decrease with the energy.

On the left side of the stability diagram, i.e., when $\kappa>0$, the stability limit is set by the radial $\pi$-mode stop-band resonance, given that the tunes increase with the energy.

Note that a second stability island exists for larger $k$ values [29]. However, we restrict our analysis to a KURNStype FFA for which the design value of $k \approx 7.6$.

Now, calculating the rms tune variations shows that the latter exhibit the expected behavior in the vicinity of the line $k_{F}=k_{D}$ where they become negligible. This is shown in Fig. 16. When field imperfections such that $\kappa \neq 0$ are

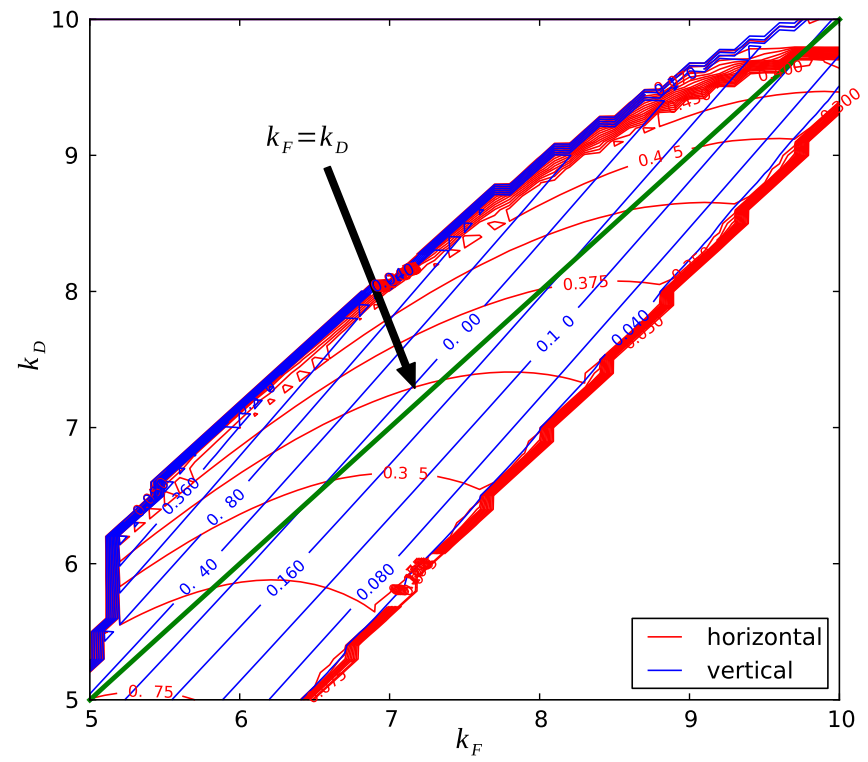

FIG. 15. Stability diagram of the average cell tune as a function of the average field index $k_{F}$ and $k_{D}$ : The green line corresponds to the case of a scaling FFA with no field imperfections, i.e., $\kappa=0$.

introduced, we can observe that the tune variations increase with $|\kappa|$ as demonstrated earlier.

Based on all the above, we compare the tracking results with those obtained from the analytical formula established in the previous section. This is shown in Figs. 17 and 18 for the horizontal and vertical plane, respectively. The red points are the simulation results, while the blue points

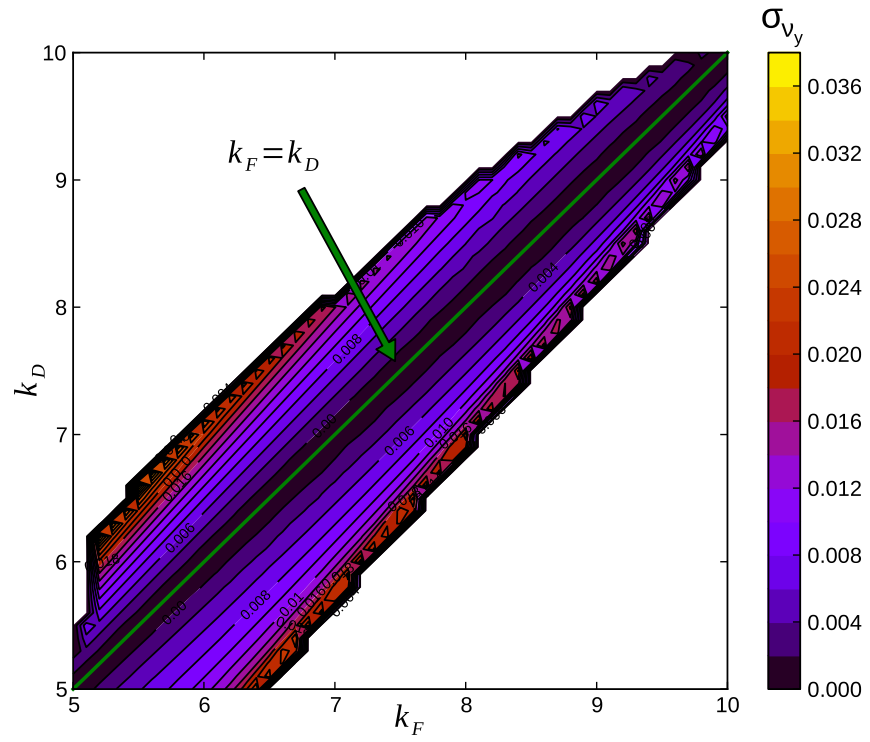

FIG. 16. Stability diagram of the rms vertical cell tune as a function of the average field index $k_{F}$ and $k_{D}$ : The green line corresponds to the case of a scaling FFA with no field imperfections, i.e., $\kappa=0$. Note that a similar result is obtained for the horizontal plane. 


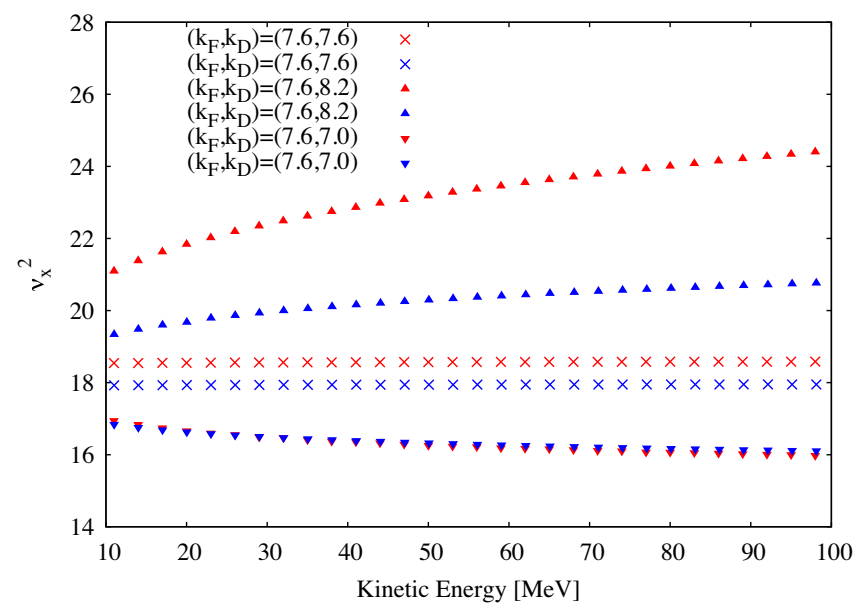

FIG. 17. Example of tune calculation using the tracking code ZGOUBI (red) and comparison with the third-order analytical formula (43) (blue). For the case $\left(k_{F}, k_{D}\right)=(7.6,8.2)$, the large discrepancy observed $(\lesssim 13 \%)$ is due to the fact that the beam is located near the boundary of stability.

represent the analytical formula: For $\kappa>0$ (upward-pointing triangle), the tunes increase with the energy in both planes, while for $\kappa<0$ (down-pointing triangle), the tunes exhibit the opposite behavior. This confirms the findings of the previous section.

The main finding of the smooth approximation is that scaling imperfections produce an orbit distortion that manifests through a radial dependence of the scalloping angle of the orbits as well as the magnetic flutter. The tracking simulations confirm our findings: As shown in Fig. 19, the Thomas focusing explains the monotonic behavior of the vertical tune as a function of the energy for various $\kappa$ values. Nevertheless, in the horizontal plane, the horizontal restoring force and the Thomas defocusing

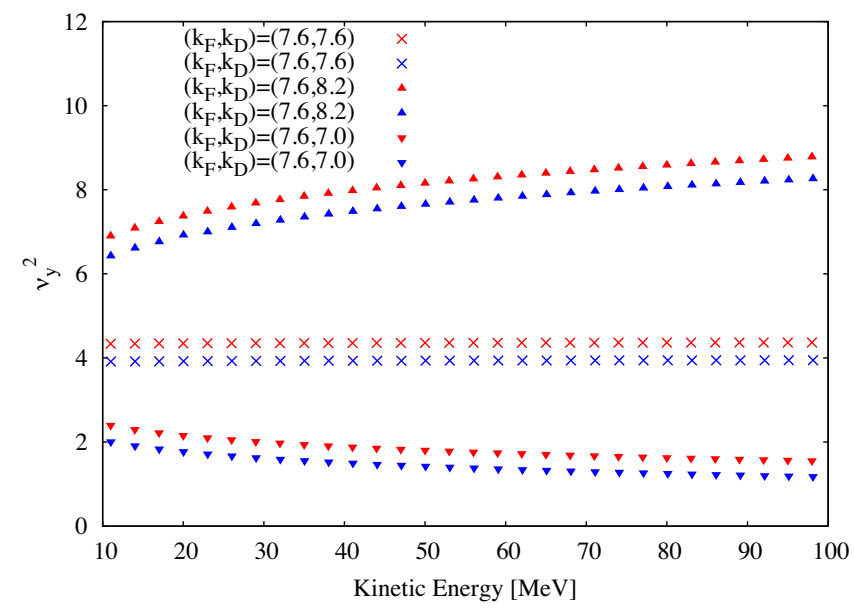

FIG. 18. Example of tune calculation using the tracking code ZGOUBI (red) and comparison with the third-order analytical formula (43) (blue). To the third order, good agreement $(<10 \%)$ is obtained in all cases considered.

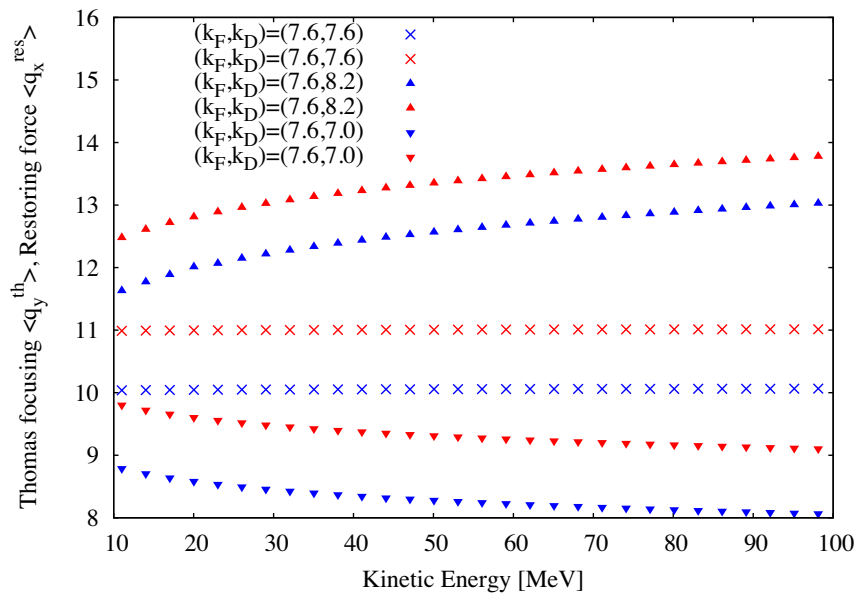

FIG. 19. The horizontal restoring force of the closed orbits (in red) as well as the Thomas focusing (in blue) as a function of the kinetic energy.

effect act in opposition such that Eq. (57) holds. Thus, it is the AG that explains the monotonic behavior of the tunes in the horizontal plane (the average field index $k$ of the orbits changes insignificantly).

\section{E. Application to the KURNS $150 \mathrm{MeV}$ scaling FFA}

We will benchmark the analytical formula against the simulated values obtained from particle tracking using 3D field maps and compare with the measurement.

The analytical model of the $D F D$ triplet is characterized by an average field index which is assumed to be constant, $k_{F}=7.6$ and $k_{D}=9.0$. The flutter function $F$ is determined at injection as shown in Fig. 6 and recalculated at higher energies by making use of Eq. (74).

For each closed orbit, the scalloping angle is solved using the fourth-order approximation and is in good agreement with the tracking results as is shown in Fig. 27. The latter are exploited to calculate the horizontal

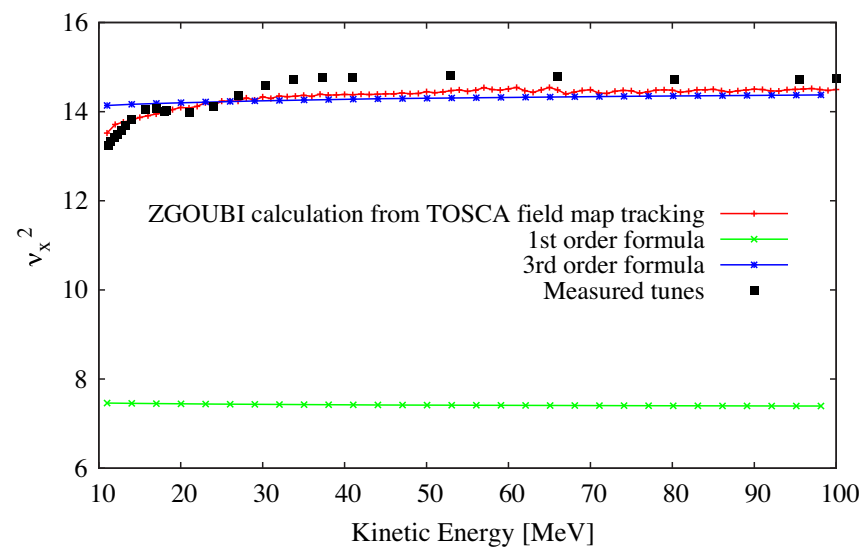

FIG. 20. Tune calculation in the horizontal plane of the KURNS $150 \mathrm{MeV}$ FFA ring and comparison with the analytical formula as well as the measurement. 


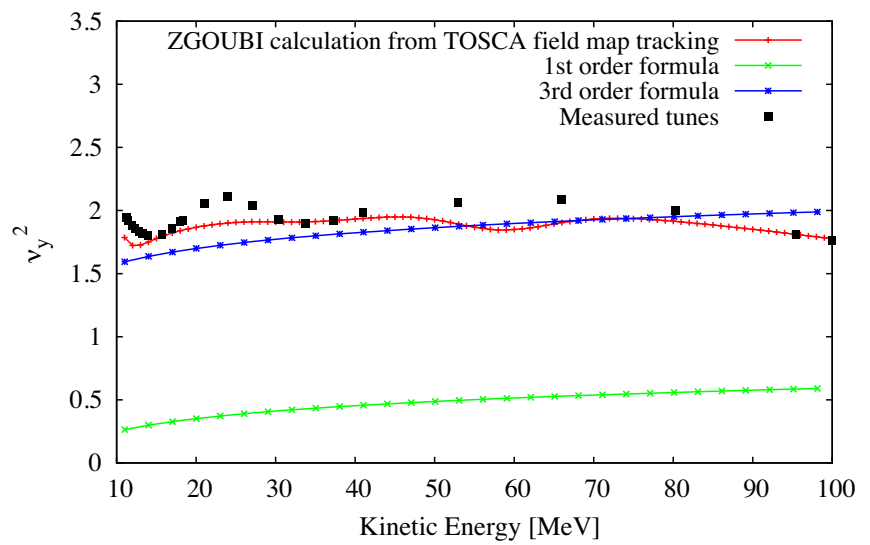

FIG. 21. Tune calculation in the vertical plane of the KURNS $150 \mathrm{MeV}$ FFA ring and comparison with the analytical formula as well as the measurement.

and vertical tunes by applying the first- and third-order approximation given by Eq. (43). The difference between the two approximations lies in the fact that the AG effect is accounted for by the higher-order terms which represent approximately half the focusing in both planes as illustrated in Figs. 20 and 21. In addition, it is important to note that the monotonic behavior of the tunes is a consequence of several localized imperfections that our simplified model does not take into account. In particular, due to the variations of the flutter function with the radius, it may be interesting to calculate the spiral focusing term to determine its impact on the beam dynamics. However, this is not what we seek in this model, which aims at simplifying the conception of the imperfections at the KURNS FFA and yields satisfactory results so far.

\section{CORRECTION SCHEME AND ADVANCED FFA CONCEPT}

Practically, it is difficult to correct the orbit and optics distortion in fixed field accelerators for the entire momentum range, since the beam moves outward radially during acceleration. Therefore, a dedicated correction system should be implemented along the radius of the magnet to produce the desired field profile. From the point of view of cyclotrons, this consists in the implementation of trim coils to correct the isochronism, i.e., the revolution time of the orbits. From the point of view of a scaling FFA, the main target is to fix the betatron wave number in both planes, which will allow one to avoid the crossing of transverse resonances and maximize the overall beam transmission from injection to extraction.

From what preceded, we obtained general rules to explain the monotonic behavior of the tunes as a function of the energy as well as the amplitude of its variations. This is a crucial result if we aim to reduce the tune excursion. One major outcome of this study is that gradient errors in the FFA magnet yield a nonscaling of the orbits and lead to a change of the average as well as the alternating gradient focusing forces applied on the beam. This means that fixing the field defect of the FFA magnet by aiming to produce a constant average field index $k$ (by considering the average field over the entire circumference) is not sufficient, since the azimuthal variations of this quantity yield a nonscaling of the orbits. In what follows, we present a novel scheme to correct the field errors in FFA that relaxes the constraint of having the scaling law valid everywhere in the ring.

\section{A. Alternating scaling imperfections}

In the context of the present study and for the sake of simplicity, the field defect is due to one of the magnets, either the focusing $(F)$ or the defocusing $(D)$ one such that $\kappa \neq 0$. Without any loss of generality, we assume that the $D$ magnet is the source of imperfection. In order to minimize the tune variations, one way is to reduce the $F D$ ratio of the $D F D$ triplet. This can be achieved by reducing its excitation current or by sliding it to outer radii so that the average field encountered by the particle at any radius is lower. However, this approach leads to the loss of focusing in both planes as shown earlier (see Fig. 14). Another interesting but not yet evaluated approach consists in misaligning vertically some of the magnets in order to modify the shape of the closed orbits. However, this is not considered in the present paper, since the changes in the vertical direction were neglected in Eq. (7). Based on the property established earlier, the tune variation with the energy exhibits antagonistic behavior based on the sign of $\kappa$. Therefore, the idea of the following correction scheme is to introduce a perturbation of the field every $P$ sectors in order to counteract the already existing imperfections. For instance, if we choose $P=2$, then a 12 -fold symmetry machine is replaced by a sixfold symmetry in the following way: Let us note $D_{i}$ (respectively, $F_{i}$ ) the defocusing (respectively, focusing) magnet with scaling factor $k_{D i}$ (respectively, $\left.k_{F i}\right)$. The original design $12 \times\left(D_{0} F_{0} D_{0}\right)$ is replaced by $6 \times\left(D_{0} F_{0} D_{0} D_{1} F_{0} D_{1}\right)$ in the following way: If $k_{D 0}>k_{F 0}$, then $k_{D 1}<k_{F 0}$ and vice versa. Thus, instead of aiming to fix the design imperfections by correcting the field profile to match with the ideal one for every magnet and make the orbits scale at every azimuthal position, the scaling of the orbits can be fixed on an average sense by creating an alternation of the difference of the average field index of the magnets. This has a major advantage of reducing the cost of the correction system, since then only $12 D$ magnets (out of 24 magnets) will require trimming coils to be implemented. The number can be further reduced if increasing $P$. This is the cornerstone of the fixed tune nonlinear nonscaling radial sector FFA concept that is discussed in detail in what follows.

\section{B. Fixed tune nonscaling FFA}

In what follows, we shall examine the characteristics of this concept which incorporates the alternation of the 


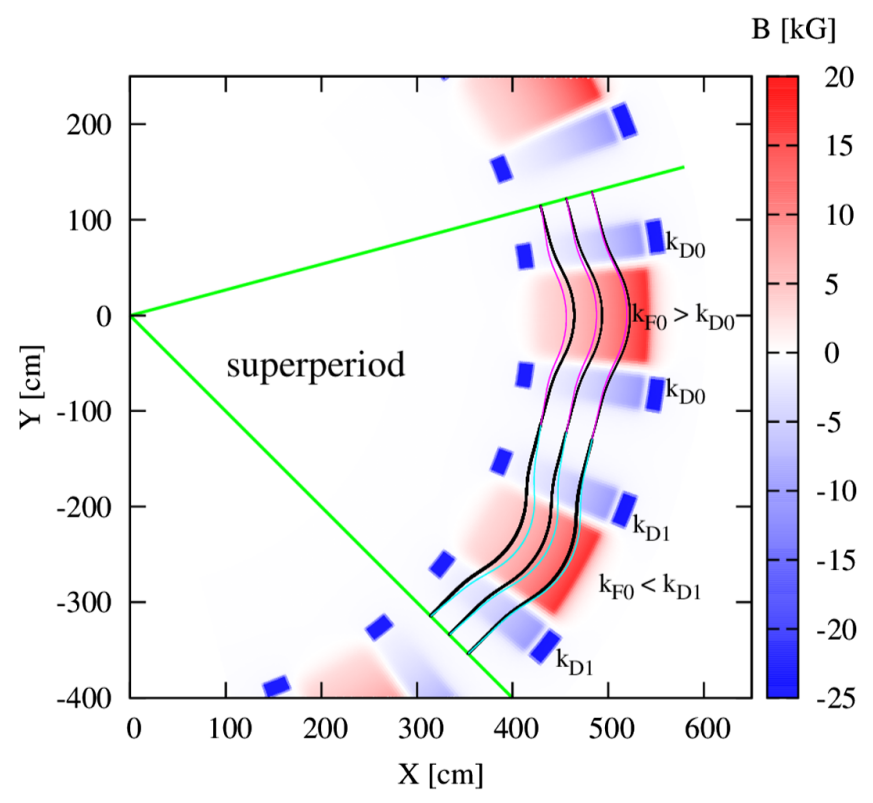

FIG. 22. Closed orbits in a scaling FFA (black) and in a fixed tune nonscaling FFA with alternating $\kappa$ (pink and light blue). For the sake of clarity, the distance between the closed orbits of the scaling and the nonscaling FFA is amplified.

difference of the gradients scheme to FFA. The particle trajectories in the median plane are shown in Fig. 22, where the orbits of a 12-fold symmetry scaling FFA $12 \times\left(k_{F 0}, k_{F 0}\right)$ are compared with those of a sixfold

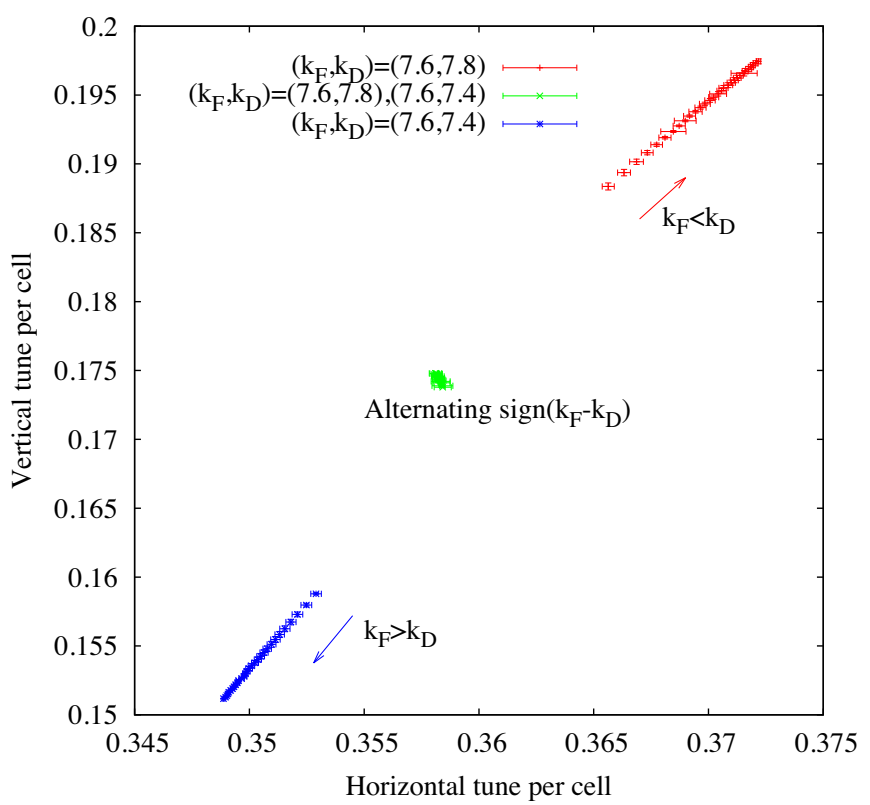

FIG. 23. Tune variations as a function of the energy before and after correction: The corrected scheme is shown in green where $\left(k_{F 0}, k_{D 0}\right)=(7.6,7.8)$ and $\left(k_{F 1}, k_{D 1}\right)=(7.6,7.4)$. These results are obtained from multiparticle tracking assuming a Gaussian distribution of the particles in the transverse plane. The error bars represent the overall tune variation from the distribution.

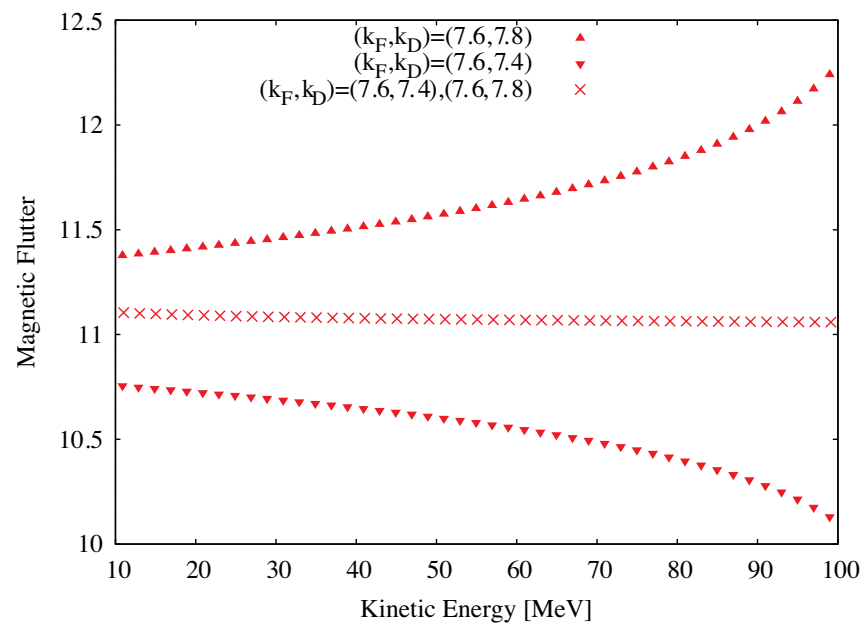

FIG. 24. Magnetic flutter in the advanced fixed tune FFA concept.

symmetry concept $6 \times\left(k_{F 0}, k_{D 0}\right),\left(k_{F 0}, k_{D 1}\right)$ : The orbit scalloping in the nonscaling case differs mainly in the dominant $F$ magnet. If $\kappa<0$, the radial field increase in the $F$ magnet is faster than in the $D$ magnet so that the equilibrium orbit (pink) in the $F$ magnet is at lower radii compared to the scaling case. The opposite effect occurs when $\kappa>0$ (light blue orbit). As a consequence of the alternation of $\kappa$, the monotonic behavior of the phase advance per cell is alternating (increasing if $\kappa>0$ and decreasing if $\kappa<0$ ). This is illustrated in Fig. 23, where the combination of two cells yields an overall fixed average tune per cell. This results from the alternation of the monotonic behavior of the horizontal restoring force as well as the Thomas focusing and the AG effect which is due to the azimuthal change of the orbit scalloping angle $\phi$. The Thomas focusing term $\left\langle q_{y}^{\text {th }}\right\rangle$ within each cell as well as for their combination is shown in Fig. 24 and is in agreement with the property established above. The same holds for all other quantities.

\section{Dynamic acceptance}

Although strong nonlinearities of the field are inherent to the scaling FFA, large dynamic acceptance (DA) is obtained with this concept. Therefore, one main question to answer is how the DA of the fixed tune nonscaling FFA compares to that of the scaling FFA.

In our analysis, the DA is defined as the maximum transverse invariant value that the beam can have without loss due to single-particle dynamics effects. Particle tracking at a fixed energy is employed for our analysis. A particle with an original displacement from the closed orbit is defined as stable if it survives 1000 turns. Given that the vertical aperture in fixed field accelerators is the limiting factor due to the small gap size, we focus our analysis on the horizontal plane. However, in our simulations, it is noted that a vertical beam size up to $1 \mathrm{~cm}$ at 


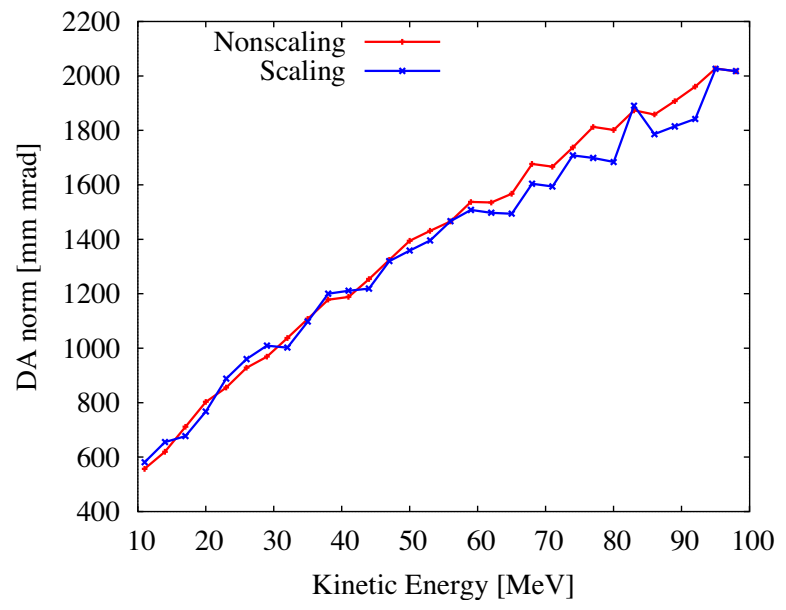

FIG. 25. Comparison of the DA of the scaling and the nonscaling FFA in the horizontal plane.

injection can be transported without any losses and that the horizontal DA is insensitive to it. The main idea is to generate two lattices that have the same tunes in both planes. This is obtained by first generating a nonscaling fixed tune lattice with $\kappa \approx 0.3$ and then matching its tunes by finely tuning the average field index as well as the $F D$ ratio of the scaling lattice. This is achieved for a lattice with $\left(\nu_{x}, \nu_{y}\right)=(4.43,2.16)$.

Comparison of the calculated DA in both cases shows (Fig. 25) that, for the same tunes, the horizontal beam acceptance is the same even though the orbits do not scale. This is valid only if resonance crossing is not occurring: In the nonscaling case discussed here, and for $P=2$, the resonance population is doubled in comparison with the scaling case. Thus, we can expect that the resonance crossing problem is more severe in the nonscaling case. This requires further investigation. Comparison of the phase space trajectories between the two cases is finally

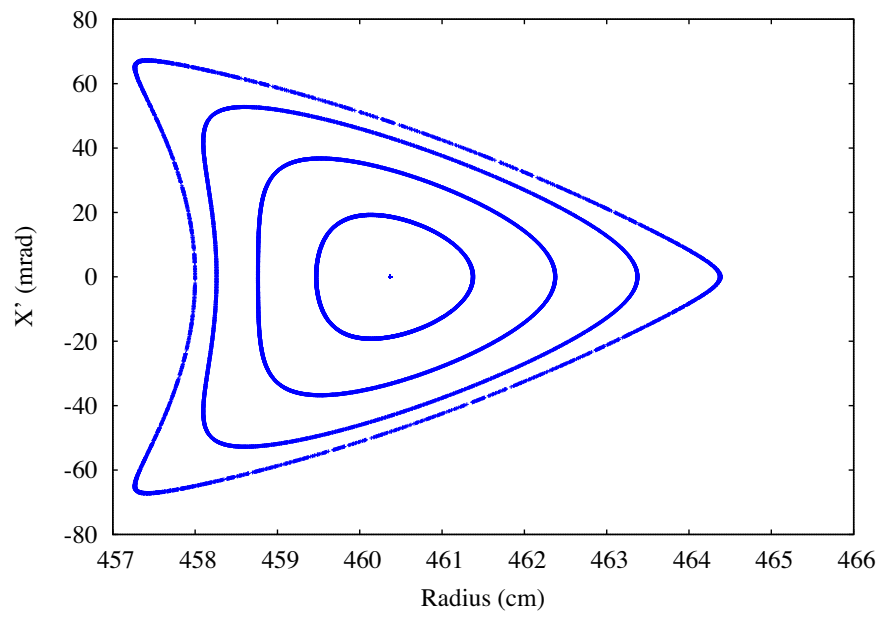

(a) shown in Fig. 26. The trajectories in both cells are symmetric with respect to $X^{\prime}=0$.

\section{CONCLUSION}

In this paper, we analyzed the stability of the particle trajectories due to field errors. Several approaches to the problem were developed and analyzed. Comparison of the results showed that the first-order approximation based on the smooth approximation is sufficient only for a lattice where the average field index is negligible. Relying on the nonlinear approach based on tracking simulations alongside the analytical derivations based on the third-order approximation from the smooth approximation, a crucial result was to establish a relationship between the betatron wave number and the field defects. A key parameter to measure the amplitude of the defects is the $\kappa$ value defined as the difference of the average field index of the focusing and defocusing magnets. Furthermore, analysis of the stability diagram (Fig. 15) showed that the tolerance to scaling imperfections becomes lower when increasing the average field index of the magnets. Based on these results, a new scheme to remediate the variation of the betatron oscillations with the energy was proposed. The main idea consists in alternating the $\kappa$ values of the magnets, every two (or more) sectors. This leads to the new concept of the fixed tune nonscaling radial sector FFA that we developed in Sec. VI: In addition to the fact that this demonstrates that the conditions of scaling are not necessary to obtain a fixed tune FFA, the newly developed concept is easier to implement by means of trim coils that can be adjusted to find the condition of minimum tune excursion and avoid the crossing of harmful resonances. Analysis of the DA showed that the lattice with alternating $\kappa$ values has the same DA as the equivalent scaling FFA case. Given that the alternating- $\kappa$ FFA reduces the number of superperiods in

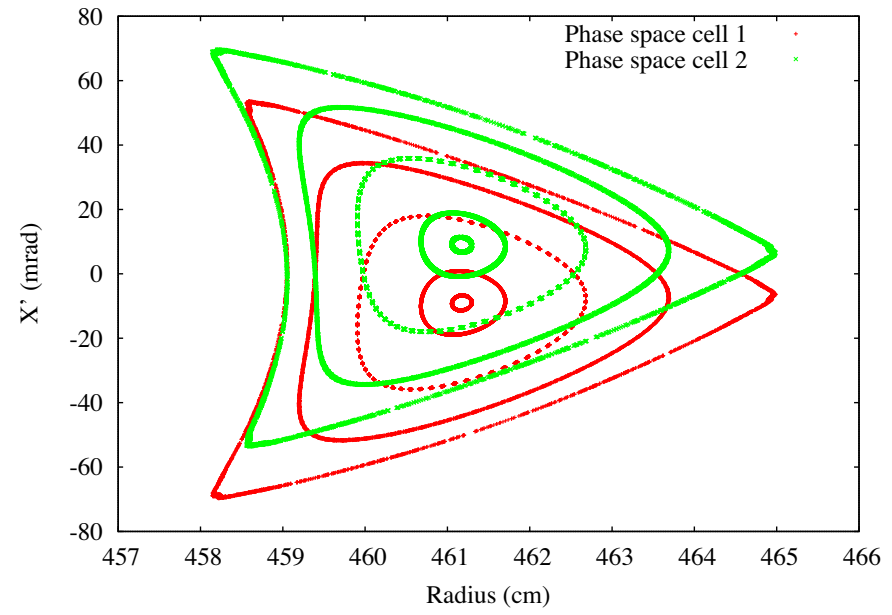

(b)

FIG. 26. Horizontal phase space trajectories at $100 \mathrm{MeV}$ including the separatrix. (a) Case of scaling FFA, (b) Case of nonscaling FFA: the trajectories in the $1^{\text {st }}$ cell are shown in red while the trajectories in the $2^{\text {nd }}$ cell areshown in green. 
the accelerator, therefore doubling the resonance population, it can be expected that the impact of the resonance crossing is more severe than the scaling FFA case. This needs further investigation.

\section{ACKNOWLEDGMENTS}

The authors express their special gratitude to the members of the international Kyoto University Research Reactor Institute Collaboration. The research leading to these results has received funding from Brookhaven Science Associates, LLC under Contract No. DE-SC0012704 with the U.S. Department of Energy.

\section{APPENDIX A: HIGHER-ORDER APPROXIMATIONS OF THE CLOSED ORBIT SCALLOPING ANGLE}

In this Appendix, we establish an improved approximation of the orbit scalloping angle. The main idea is to use the method of successive approximations for the set of coupled equations (2) and (41) in order to obtain higherorder approximations of the scalloping angle of the closed orbit.

First, Eq. (2) can be integrated, and the radius of the closed orbit is given by

$$
R(\theta)=R(0) \exp \left(-\int_{0}^{\theta} \tan [\phi(\theta)] d \theta\right) .
$$

Now, from Eq. (41), the expression of the scalloping angle can be rewritten in the general form:

$$
1+\dot{\phi}=\frac{B_{m}[R(\theta)] \cdot R(\theta) \cdot F(\theta) / \cos (\phi)}{\left\langle B_{m}[R(\theta)] \cdot R(\theta) \cdot F(\theta) / \cos (\phi)\right\rangle},
$$

where the condition of the closed path of the orbit is satisfied by ensuring that $\langle 1+\dot{\phi}\rangle=1$. Thus, solving Eqs. (A1) and (A2) simultaneously shall yield the exact scalloping angle of

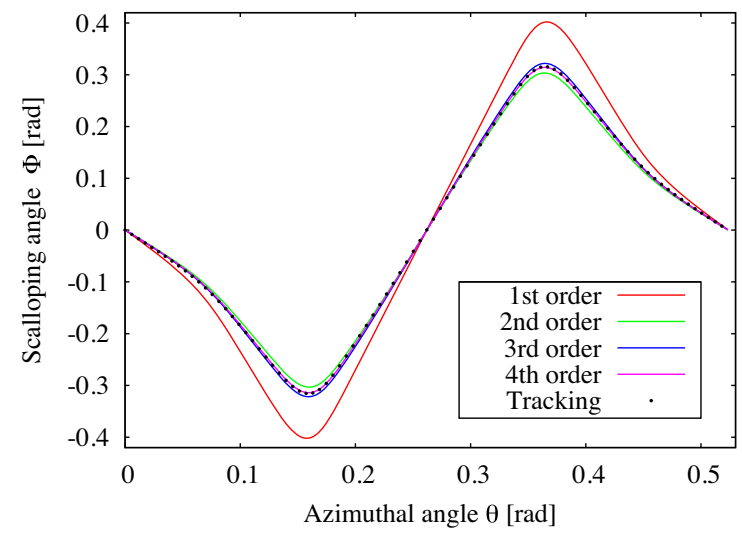

the median plane closed orbit. For that, the nature of the function $B_{m}(R)$ describing the evolution of the average magnetic field with the radius must be taken into account in order to obtain higher-order approximations.

Since the focus of our analysis in the present paper is on nearly scaling FFA, we will assume that $B_{m}(R)$ obeys the $R^{k}$ law. Injecting the first-order approximation $\phi \approx \tilde{F}$ into the right-hand-side term of Eq. (A2) yields

$$
\begin{aligned}
1+\dot{\phi} & \approx \frac{\exp \left\{-(k+1) \int_{0}^{\theta} \tan [\tilde{F}(\theta)] d \theta\right\}}{\left\langle\exp \left\{-(k+1) \int_{0}^{\theta} \tan [\tilde{F}(\theta)] d \theta\right\} \cdot F(\theta)\right\rangle} F(\theta) \\
& =h(k, \theta) F(\theta),
\end{aligned}
$$

where the tilde operator is the integrating operator defined by $\tilde{F}(\theta)=\int_{0}^{\theta}(F-\langle F\rangle) d \theta$. This shows that the scalloping angle is a function of the average field index of the magnet as well as the flutter function $F$ [note that the impact of the cosine function in Eq. (A2) can be neglected for the secondorder approximation - it plays a role in improving the approximation at a higher order]. To our knowledge, this is the first time that such a relationship is established analytically in FFA. In particular, this demonstrates that, for radial sector cyclotrons, the orbits do not scale, since $k$ is a function of the radius. To proceed with the approximation to higher orders, the idea is to reinject the newly obtained approximation $\phi(k, \theta) \approx h(\widehat{k, \theta) F}(\theta)$ into Eqs. (A1) and (A2) consecutively and then integrate. The newly obtained approximations will be in the same form.

The convergence of this iterative scheme is shown in Fig. 27, where we compared several high-order approximations with the results of tracking. Usually, the secondorder approximation is sufficient to obtain an accurate calculation of the shape of the equilibrium orbit with a relative error below $5 \%$.

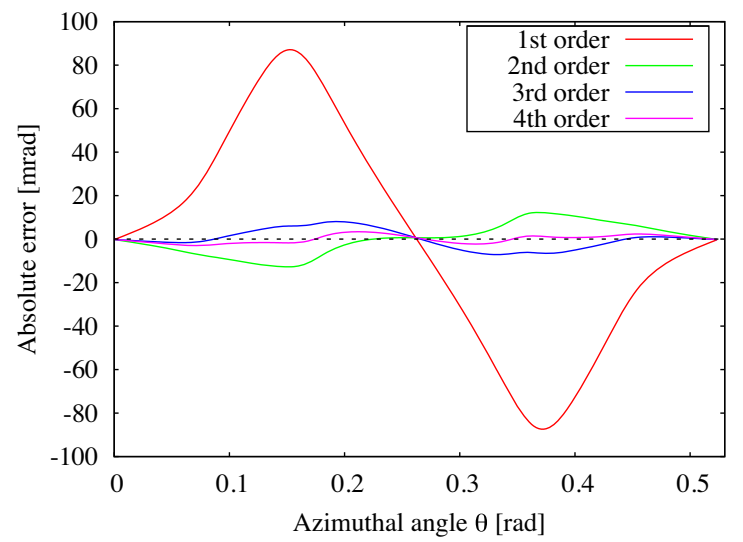

FIG. 27. Comparison of the analytical approximation of the orbit scalloping angle with the tracking results based on the 3D field map of the KURNS FFA: The second-order approximation yields an absolute error below 12 mrad, while the fourth-order approximation reduces the absolute error below $3 \mathrm{mrad}$. 


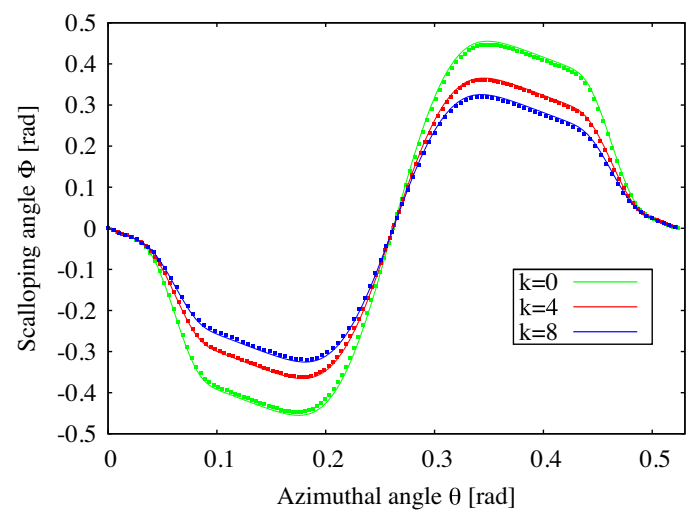

FIG. 28. Comparison of the tracking results (dots) with the analytical estimates based on the second-order approximation (solid lines): For all cases considered, the absolute error is below 10 mrad.

Note in addition that, in the limit where $k \rightarrow 0$ and $F(\theta) \rightarrow 1, h(k, \theta) \rightarrow 1$ so that the first-order approximation is sufficient. Taking the partial derivative of the $h$ function yields

$$
\frac{\partial h}{\partial \theta}=-(k+1) \tan [\tilde{F}(\theta)] h
$$

Finally, to illustrate the impact of the average field index on the scalloping angle of the closed orbit, we assume a lattice for which the flutter function is fixed and vary the $k$ values. The tracking results are compared with the analytical estimates (solid lines) in Fig. 28, where a good agreement on the $5 \%$ level is achieved. This shows that the orbit scalloping decreases with increasing $k$ and, hence, the edge focusing decreases as well as shown in Sec. IV E.

\section{APPENDIX B: ANALYTICAL EXPRESSION OF THE MAGNETIC FIELD TO ACCOUNT FOR RADIAL DEFECTS}

In order to obtain the radial dependence of the field when the mean field index $k$ is $R$ dependent, let us assume that $k$ can be fitted with an $n$-order polynomial. Thus, $k$ is written in the following way:

$$
k(R)=\sum_{i=0}^{n} a_{i}\left(\frac{R}{R_{0}}\right)^{i}
$$

Then equating Eqs. (70) and (B1) yields

$$
\frac{d B}{B}=\sum_{i=0}^{n} \frac{a_{i}}{R_{0}^{i}} R^{i-1} d R=a_{0} \frac{d R}{R}+\sum_{i=1}^{n} \frac{a_{i}}{R_{0}^{i}} R^{i-1} d R,
$$

which gives after integration

$$
B(R)=B_{0} \exp \left[a_{0} \ln \left(\frac{R}{R_{0}}\right)+\sum_{i=1}^{n} \frac{a_{i}}{R_{0}^{i}} \frac{\left(R^{i}-R_{0}^{i}\right)}{i}\right]
$$

so that the general form of the magnetic field becomes $B(R, \theta)=B(R) F(\theta)$, i.e.,

$$
B(R, \theta)=B_{0}\left(\frac{R}{R_{0}}\right)^{a_{0}} \times \exp \left(\sum_{i=1}^{n} a_{i} \frac{R^{i}-R_{0}^{i}}{i \times R_{0}^{i}}\right) \times F(\theta) .
$$

[1] K. R. Symon, D. W. Kerst, L. W. Jones, L. J. Laslett, and K. M. Terwilliger, Fixed-Field Alternating-Gradient Particle Accelerators, Phys. Rev. 103, 1837 (1956).

[2] T. Ohkawa, Two-beam fixed field alternating gradient accelerator, Rev. Sci. Instrum. 29, 108 (1958).

[3] A. A. Kolomensky and A. N. Lebedev, Theory of Cyclic Accelerators (North-Holland, Amsterdam, 1966), pp. 77-81.

[4] The name of the institute has been changed since April 2018. It used to be Kyoto University Research Reactor Institute (KURRI).

[5] C. H. Pyeon et al., First injection of spallation neutrons generated by high-energy protons into the Kyoto University critical assembly, J. Nucl. Sci. Technol. 46, 1091 (2009).

[6] Y. Ishi, Status of KURRI Facility, in Proceedings of the FFAG 2016 workshop, Imperial college, London, 2016, https://indico.cern.ch/event/543264/contributions/ 2295846/attachments/1333675/2005286/FFAG16_ LONDON_ishi.pdf.

[7] S. L. Sheehy et al., Characterization techniques for fixed field alternating gradient accelerators and beam studies using the $150 \mathrm{MeV}$ proton FFAG, Prog. Theor. Exp. Phys. (2016), $073 \mathrm{G} 01$.

[8] S. Machida et al., Acceleration in the linear non-scaling fixed-field alternating-gradient accelerator EMMA, Nat. Phys. 8, 243 (2012).

[9] D. Trbojevic et al., CBETA-Cornell University Brookhaven National Laboratory Electron Energy Recovery Test Accelerator, in Proceedings of IPAC'17, Copenhagen, Denmark (2017), TUOCB3, https://doi.org/10.18429/ JACoW-IPAC2017-TUOCB3.

[10] E. C. Aschenauer et al., eRHIC Design Study: An electronIon Collider at BNL, arXiv:1409.1633.

[11] F. Méot, Zgoubi users guide, Report No. CA/AP/470, BNL C-AD, 2012.

[12] E. Courant and H. Snyder, Theory of the alternatinggradient synchrotron, Ann. Phys. (Paris) 3, 1 (1958).

[13] F. T. Cole, R. O. Haxby, L. W. Jones, C. H. Pruett, and K. M. Terwilliger, Electron model fixed field alternating gradient accelerator, Rev. Sci. Instrum. 28, 403 (1957).

[14] S. Sheehy, K. Peach, H. Witte, D. J. Kelliher, and S. Machida, Fixed field alternating gradient accelerator with small orbit shift and tune excursion, Phys. Rev. Accel. Beams 13, 040101 (2010).

[15] K. J. Peach et al., Conceptual design of a non-scaling fixed field alternating gradient accelerator for protons and carbon 
ions for charged particle therapy, Phys. Rev. Accel. Beams 16, 030101 (2013).

[16] L. C. Teng, The smooth approximation to Hill equation, Report No. ANL-1557, 1956.

[17] S. Machida, Resonance crossing and dynamic aperture in nonscaling fixed field alternating gradient accelerators, Phys. Rev. Accel. Beams 11, 094003 (2008).

[18] M. Craddock and K. R. Symon, Cyclotrons and fixed-field alternating-gradient accelerators, Rev. Accel. Sci. Techol. 01, 65 (2008).

[19] J. J. Livingood, Principles of Cyclic Particle Accelerators (Van Nostrand, Princeton, 1961).

[20] Y. Jongen and S. Zaremba, Cyclotron magnet calculations, Report No. CAS CERN 96/02, 1996.

[21] S. Zaremba and W. Kleeven, Cyclotrons magnetic design and beam dynamics, in Proceedings of CERN Accelerator School, Accelerators for Medical Applications, Vösendorf, Austria, 2015, https://doi.org/10.23730/CYRSP-2017001.177.

[22] H. L. Hagedoorn and N.F. Verster, Orbits in an AVF cyclotron, Nucl. Instrum. Methods 18-19, 201 (1962).

[23] L. H. Thomas, The paths of ions in the cyclotron I. Orbits in the magnetic field, Phys. Rev. 54, 580 (1938).

[24] H. A. Bethe and M.E. Rose, The maximum energy obtainable from the cyclotron, Phys. Rev. 52, 1254 (1937).

[25] M. Craddock, AG focusing in the Thomas cyclotron of 1938, FR5REP 113, in Proceedings of the 23rd Particle Accelerator Conference, Vancouver, Canada, 2009 (IEEE, Piscataway, NJ, 2009).

[26] T. Adachi et al., A $150 \mathrm{MeV}$ FFAG synchrotron with 'RETURN-YOKE FREE' magnet, in Proceedings of the 19th Particle Accelerator Conference, Chicago, IL, 2001 (IEEE, Piscataway, NJ, 2001), p. 3254.

[27] Y. Ishi et al., Practical betatron tune behavior during acceleration in scaling FFAG rings at KURNS, THPAK034, in Proceedings of IPAC'18, Vancouver, Canada, 2018, 10 .18429/JACoW-IPAC2018-THPAK034.

[28] S. L. Sheehy et al., Characterization of the KURRI $150 \mathrm{MeV}$ FFAG and plans for high intensity experiments, in Proceedings of the 57th ICFA Advanced Beam Dynamics Workshop on High Intensity, High Brightness and High Power Hadron Beams (HB2014), Michigan, 2014, paper MOPAB27, pp. 89-93, https://accelconf.web.cern.ch/ HB2014/papers/mopab27.pdf.

[29] S. Machida, Scaling Fixed-Field Alternating Gradient Accelerators with a Small Orbit Excursion, Phys. Rev. Lett. 103, 164801 (2009).

[30] FFAG14 Web site, https://www.bnl.gov/ffag14/, the FFA acronym has been adopted since 2017 instead of FFAG.

[31] V. Smirnov, Computer codes for beam dynamics analysis of cyclotronlike accelerators, Phys. Rev. Accel. Beams 20, 124801 (2017).

[32] M. K. Craddock and Y.-N. Rao, Orbit tracking in FFAGs and reverse-bend cyclotrons using the CYCLOPS code, in Proceedings of FFAG'08, Manchester, England, 2008, https://www.cockcroft.ac.uk/events/FFAG08/ presentations/Craddock/Orbit\%20tracking\%20talk.pdf.

[33] S. L. Sheehy et al., Progress on simulation of fixed field alternating gradient accelerators, MOPJE077, in Proceedings of IPAC'15, Virginia, 2015, https://doi.org/10.18429/ JACoW-IPAC2015-MOPJE077.

[34] H. A. Enge, in Focusing of Charged Particles, edited by A. Spetier (Academic, New York, 1967), Vol. 2, p. 203. 